The Astrophysical Journal, 486:770-783, 1997 September 10

(C) 1997. The American Astronomical Society. All rights reserved. Printed in U.S.A.

\title{
MULTIWAVELENGTH MONITORING OF THE BL LACERTAE OBJECT PKS 2155-304 IN 1994 MAY. I. THE GROUND-BASED CAMPAIGN
}

\author{
Joseph E. Pesce,${ }^{1}$ C. Megan Urry, ${ }^{1}$ Laura Maraschi, ${ }^{2}$ Aldo Treves, ${ }^{3}$ Paola Grandi, ${ }^{4}$ Ronald \\ I. Kollgaard, ${ }^{5,6}$ Elena Pian, ${ }^{1}$ Paul S. Smith,${ }^{7,8}$ Hugh D. Aller, ${ }^{9}$ Margo F. Aller,${ }^{9}$ Aaron J. \\ Barth,${ }^{10}$ David A. H. Buckley, ${ }^{11}$ Elvira Covino, ${ }^{12}$ AleXei V. Filippenko, ${ }^{10}$ Eric J. Hooper,${ }^{7}$

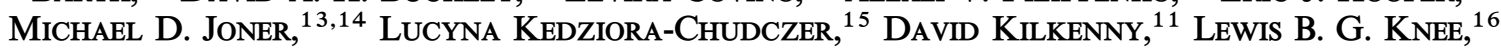 \\ Michael KunKel,${ }^{17}$ Andrew C. Layden, ${ }^{18}$ Antonio Mário Magalhães,,${ }^{19}$ Fred Marang, ${ }^{11}$ Vera \\ E. Margoniner, ${ }^{19}$ Christopher Palma,,${ }^{5,20}$ Antonio Pereyra, ${ }^{19}$ Claudia V. Rodrigues, ${ }^{19,21}$ \\ ANDRIEs Schutte, ${ }^{22}$ Michael L. SitKo, ${ }^{23}$ Merja ToRnikoski, ${ }^{24}$ Johan VAN DER Walt, ${ }^{25}$ \\ FrancoIs VAN WYK, ${ }^{11}$ Patricia A. Whitelock, ${ }^{11}$ AND SCOTT J. WOLK ${ }^{14,26}$ \\ Received 1996 July 12; accepted 1997 April 21
}

\section{ABSTRACT}

Optical, near-infrared, and radio observations of the BL Lac object PKS 2155-304 were obtained simultaneously with a continuous UV/EUV/X-ray monitoring campaign in 1994 May. Further optical observations were gathered throughout most of 1994. The radio, millimeter, and near-infrared data show no strong correlations with the higher energies. The optical light curves exhibit flickering of $0.2-0.3 \mathrm{mag}$ on timescales of 1-2 days, superposed on longer timescale variations. Rapid variations of $\sim 0.01 \mathrm{mag}$ minute $^{-1}$, if real, are the fastest seen to date for any BL Lac object. Small $(0.2-0.3 \mathrm{mag})$ increases in the $V$ and $R$ bands occur simultaneously with a flare seen at higher energies. All optical wave bands $(U B V R I)$ track each other well over the period of observation, with no detectable delay. For most of the period the average colors remain relatively constant, although there is a tendency for the colors (in particular, $B-V$ ) to vary more when the source fades. In polarized light, PKS 2155-304 showed strong color dependence (polarization increases toward the blue, $P_{U} / P_{I}=1.31$ ) and the highest optical polarization $(U=14.3 \%)$ ever observed for this source. The polarization variations trace the flares seen in the UV flux. For the fastest variability timescale observed, we estimate a central black hole mass of $\lesssim 1.5$ $\times 10^{9}(\delta / 10) M_{\odot}$, consistent with UV and X-ray constraints and smaller than previously calculated for this object.

Subject headings: BL Lacertae objects: individual (PKS 2155-304) - galaxies: active galaxies: photometry - polarization

\section{INTRODUCTION}

Among active galactic nuclei (AGNs), the blazar class (BL Lacertae objects and violently variable quasars) is known for rapid variability, high luminosity, and a high level of polarization. The observed properties of blazars are currently interpreted as nonthermal (synchrotron and inverse Compton) emission from an inhomogeneous, relativistic jet oriented close to the line of sight (Blandford \&

\footnotetext{
${ }^{1}$ Space Telescope Science Institute, 3700 San Martin Drive, Baltimore, MD 21218. The Space Telescope Science Institute is operated by the Association of Universities for Research in Astronomy, Inc., under contract with the National Aeronautics and Space Administration.

2 Osservatorio Astronomico di Brera, via Brera 28, I-20121 Milano, Italy.

${ }^{3}$ International School for Advanced Studies (SISSA), via Beirut 2-4, I-34014 Trieste, Italy; present address: Department of Physics, University of Milan at Como, via Lucini, I-22100 Como, Italy.

${ }^{4}$ Istituto di Astrofisica Spaziale, CNR, via Enrico Fermi 23, C.P. 67, I-00044 Frascati, Italy.

${ }^{5}$ Department of Astronomy and Astrophysics, Pennsylvania State University.

${ }^{6}$ Present address: Fermi National Accelerator Laboratory, P.O. Box 500, Batavia, IL 60510.

${ }^{7}$ Steward Observatory, University of Arizona, Tucson, AZ 85721.

${ }^{8}$ Present address: Kitt Peak National Observatory, NOAO, P.O. Box 26732, Tucson, AZ 85726-6732.

9 Department of Astronomy, University of Michigan, 830 Dennison, 501 East University Avenue, Ann Arbor, MI 48109-1090.

${ }^{10}$ Department of Astronomy, 601 Campbell Hall, University of California at Berkeley, Berkeley, CA 94720-3411.

${ }^{11}$ South African Astronomical Observatory, P.O. Box 9, Observatory 7935, Western Cape, South Africa.

12 Osservatorio Astronomico di Capodimonte, via Moiariello 16, I-80131 Napoli, Italy.

13 Department of Physics and Astronomy, 263 FB, Brigham Young University, Provo, UT 84602.

${ }^{14}$ Visiting Astronomer, Cerro Tololo Inter-American Observatory (CTIO), La Serena, Chile. CTIO is operated by the Association of Universities for Research in Astronomy, Inc., under contract with the National Science Foundation.

${ }_{15}$ Australia Telescope National Facility, P.O. Box 76, Epping, NSW 2121, Australia.

${ }^{16}$ Swedish-ESO Submillimetre Telescope, European Southern Observatory, Casilla 19001, Santiago 19, Chile; also Onsala Space Observatory, S-43992 Onsala, Sweden.

${ }^{17}$ Max-Planck-Institut für Astronomie, Königstuhl 17, D-69117 Heidelberg, Germany.

${ }^{18}$ Cerro Tololo Inter-American Observatory, Casilla 603, La Serena, Chile. Present address: Department of Physics and Astronomy, McMaster University, Hamilton, ON L8S 4M1, Canada.

${ }^{19}$ Instituto Astronômico e Geofisico, Universidade de São Paulo, C.P. 9638, São Paulo, SP 01065-970, Brazil.

${ }^{20}$ Present address: Department of Astronomy, University of Virginia, P.O. Box 3818, Charlottesville, VA 22903-0818.

${ }^{21}$ Present address: Divisão de Astrofisica, Instituto Nacional de Pesquisas Espaciais, C.P. 515, São José dos Campos, SP 12201-970, Brazil.

${ }^{22}$ Department of Physics, University of Zululand. Present address: Siemens Telecommunications, 270 Maggs Street, Waltloo, Pretoria, South Africa.

${ }^{23}$ Department of Physics, University of Cincinnati, Cincinnati, OH 45221-0011.

${ }^{24}$ Metsähovi Radio Research Station, Metsähovintie 114, FIN-02540 Kylmälä, Finland.

${ }^{25}$ Space Research Unit, Department of Physics, Potchefstroom University, Potchefstroom 2520, South Africa.

${ }^{26}$ Astronomy Program, Department of Earth and Space Sciences, State University of New York at Stony Brook, Stony Brook, NY 11794-2100.
} 
Rees 1978). Typical jet models (Ghisellini, Maraschi, \& Treves 1985; Marscher \& Gear 1985; Königl 1989) have a large number of free parameters and are underconstrained by single-epoch spectral distributions. Combining spectral and temporal information greatly constrains the jet physics, since different models predict different variability as a function of wavelength. Elucidating the structure of blazar jets through multiwavelength monitoring and polarization studies is an essential precursor to understanding their formation and, thus, the extraction of energy from the central engine.

At low frequencies (radio-millimeter-infrared-optical), this technique has already led to substantial progress: the evolution of radio flares in time and frequency has been used to deduce the structure of the outer parts of the jet (Hughes, Aller, \& Aller 1989). The variations among the radio bands are well correlated, and lags are typically weeks to months. In some cases, optical variations precede radio ones by about a year, although only weak correlations have been established (Bregman \& Hufnagel 1989). Some blazars also exhibit intraday variability at optical and radio wavelengths (Wagner \& Witzel 1995, and references therein). Optical polarimetry shows that the synchrotron continuum completely dominates the emission from most blazars at optical and UV wavelengths (Smith \& Sitko 1991). While variations are present at all frequencies, blazars are generally most variable at the shortest wavelengths (optical, UV, $\mathrm{X}$-ray).

The BL Lac object PKS 2155-304 is an excellent candidate for blazar monitoring because it is both rapidly variable and bright enough that its variability can be resolved at wavelengths shorter than optical (Edelson et al. 1995); in particular, PKS $2155-304$ is one of only two blazars (the other being Mrk 421) that can be monitored sufficiently rapidly with the International Ultraviolet Explorer satellite. It is also one of the brightest extragalactic sources detected with the Extreme Ultraviolet Explorer satellite (EUVE; Marshall, Carone, \& Fruscione 1993; Fruscione et al. 1994). PKS 2155-304 is one of the strongest X-ray emitters and is a typical X-ray-selected BL Lac object.

High-energy gamma rays from PKS 2155-304 have been detected recently by the EGRET experiment on board the Compton Gamma Ray Observatory (CGRO; Vestrand, Stacy, \& Sreekumar 1995), confirming that the emission processes in PKS 2155-304 are similar to those in the many blazars already detected with CGRO. Thus, by studying multiwavelength variability in this bright and highly variable object, we derive information relevant for the whole class, especially for the "high-frequency peaked BL Lacs" (Padovani \& Giommi 1995), i.e., X-ray-selected BL Lac objects.

Attempts at multiwavelength studies of PKS 2155-304 with IUE and EXOSAT (Treves et al. 1989) indicated a correlation of the two wave bands but also the need for much better sampling. Multiwavelength monitoring of PKS 2155-304 in 1991 November (Smith et al. 1992; Urry et al. 1993; Brinkmann et al. 1994; Courvoisier et al. 1995; Edelson et al. 1995) produced the best available data for any blazar. This soft X-ray/UV/optical monitoring of PKS 2155-304 found that the emission at these wavelengths was well correlated, that there was significant shorttimescale variability ( $<1$ day), and that the X-ray flux led the UV by a few hours. The tight X-ray/UV correlation and the overall UV to X-ray spectral shape confirmed the sup- position that synchrotron emission is responsible for the optical through X-ray continuum in this BL Lac object (and presumably in others with similar spectra and variability) and ruled out conclusively any observed optical/UV continuum from an accretion disk (as argued also on the basis of polarization studies in the optical/UV). However, this campaign had sufficient sampling only over a short period of time (4 days).

For this reason, a second campaign was organized in 1994 May in which the intensive IUE monitoring was extended to 10 days. The UV, extreme-ultraviolet, and $\mathrm{X}$-ray observations, as well as the overall multiwavelength campaign, are addressed elsewhere (Pian et al. 1997; Marshall et al. 1997; Kii et al. 1997; Urry et al. 1997). Here we discuss the ground-based observations during the 1994 May campaign and beyond.

This paper is organized as follows: In $\S 2$, we present the ground-based observations made from 1994 May through 1994 November. Section 3 includes a discussion of these data, and conclusions are given in $\S 4$.

\section{MULTIWAVELENGTH GROUND-BASED OBSERVATIONS}

\subsection{Radio}

The Very Large Array (VLA) ${ }^{27}$ was used in a hybrid A/B configuration to monitor the arcsecond core of PKS 2155-304 on 12 days (1994 May 14-June 1) at three frequencies $(8.4,15.0$, and $22.5 \mathrm{GHz})$, with 1.5 and $5.0 \mathrm{GHz}$ measurements also taken on four of these occasions (see Table 1). Standard frequency settings and dual $50 \mathrm{MHz}$ bandwidths were used. The uncertainties listed in the table are the internal errors and do not include systematic effects, which will alter the overall flux scale (see below).

Observations of a few minutes were made at each frequency with similar but not identical $u$-v coverage on the different days. Complementary observations (once per day at each frequency) were also made of $3 \mathrm{C} 48$ and one or both of two nearby calibrator sources (HB89 2149-307; HB89 $2245-328$ ), which we assumed to be nonvariable. Because of poor $u-v$ coverage of $3 C 48$, standard VLA calibration techniques failed, and we determined the flux densities directly from the raw $u-v$ data. The absolute flux scale is therefore dependent upon the overall gain normalization applied to the data during calibration and should be accurate to $10 \%$ at $22.5 \mathrm{GHz}$ and $5 \%$ at the four lower frequencies (R. A. Perley, 1994, private communication). However, the flux densities obtained from 3C 48 and the calibrator sources show that the relative flux scale is better than this, with variations of $1 \%, 1 \%, 3 \%, 3 \%$, and $4 \%$ noted at $1.5,5,8.4,15$, and $22.5 \mathrm{GHz}$, respectively. At all frequencies PKS 2155-304 was more variable than the calibrator sources. The data collected on May 26 (MJD $9,499.05)^{28}$ were systematically low for all sources and have been scaled by assuming that the calibrators are nonvariable.

Data at three frequencies $(4.8,8$, and $14.5 \mathrm{GHz})$ were also obtained with the University of Michigan Radio Astronomy Observatory (UMRAO) $26 \mathrm{~m}$ single-dish telescope (Table 2). The observational technique and reduction procedures are discussed by Aller et al. (1985). Typically, each daily observation consists of a series of on-off measure-

\footnotetext{
27 The National Radio Astronomy Observatory is operated by Associated Universities, Inc., under cooperative agreement with the NSF.

${ }^{28}$ In this paper, MJD is defined as JD - 2,440,000.
} 
TABLE 1

VLA RADio DATA

\begin{tabular}{|c|c|c|c|c|c|c|}
\hline \multirow{2}{*}{$\begin{array}{c}\text { DATE OBSERVED } \\
\text { (1994) }\end{array}$} & \multirow{2}{*}{$\begin{array}{c}\text { JD } \\
(-2,440,000)\end{array}$} & \multicolumn{5}{|c|}{ FLux (mJy) } \\
\hline & & $1.5 \mathrm{GHz}$ & $5.0 \mathrm{GHz}$ & $8.4 \mathrm{GHz}$ & $15.0 \mathrm{GHz}$ & $22.5 \mathrm{GHz}$ \\
\hline May 14 & $9,487.04$ & & & $465+1$ & $499+1$ & $488+3$ \\
\hline May $15 \ldots \ldots \ldots$ & $9,488.13$ & $419 \pm 1$ & $509 \pm 1$ & $451 \pm 1$ & $504 \pm 1$ & $476 \pm 2$ \\
\hline May 16 .......... & $9,489.08$ & $-2=-1$ & -2 & $465+1$ & $506 \pm 1$ & $481 \pm 2$ \\
\hline May $17 \ldots \ldots \ldots$ & $9,490.08$ & $\ldots$ & $\ldots$ & $463 \pm 1$ & $502 \pm 1$ & $547 \pm 2$ \\
\hline May $18 \ldots \ldots \ldots$ & $9,491.07$ & $\ldots$ & $\ldots$ & $466 \pm 1$ & $500 \pm 1$ & $527 \pm 2$ \\
\hline May $21 \ldots \ldots \ldots$ & $9,494.10$ & $365 \pm 1$ & $515 \pm 1$ & $488 \pm 1$ & $523 \pm 1$ & $556 \pm 2$ \\
\hline May 22 . & $9,495.06$ & $\ldots$ & $\ldots$ & $498 \pm 1$ & $542 \pm 1$ & $536 \pm 2$ \\
\hline May 23 .......... & $9,496.09$ & $\ldots$ & $\ldots$ & $508 \pm 1$ & $534 \pm 1$ & $524 \pm 3$ \\
\hline May $24 \ldots$. & $9,497.03$ & $\ldots$ & $\ldots$ & $509 \pm 1$ & $556 \pm 1$ & $524 \pm 2$ \\
\hline May $26 \ldots \ldots \ldots$ & $9,499.05$ & $\ldots$ & $\ldots$ & $481 \pm 1$ & $549 \pm 2$ & $632 \pm 3$ \\
\hline May $28 \ldots \ldots \ldots$ & $9,501.05$ & $395 \pm 1$ & $537 \pm 1$ & $486 \pm 2$ & $545 \pm 1$ & $517 \pm 3$ \\
\hline Jun $1 \ldots \ldots \ldots \ldots$ & $9,505.04$ & $392 \pm 1$ & $514 \pm 1$ & $491 \pm 1$ & $505 \pm 1$ & $473 \pm 2$ \\
\hline
\end{tabular}

Notes.-VLA data from R. I. K. and C. P. Errors shown are the internal errors. Total uncertainties are $\sim 10 \%$, as in Fig. 1 .

ments over a $30-45$ minute time period. The flux scale is based on observations of 3C 461 and the absolute scale of Baars et al. (1977). This primary standard, or a nearby secondary flux standard (one of 3C 58, 3C 144, 3C 145, 3C 218, $3 \mathrm{C} 274,3 \mathrm{C} 286,3 \mathrm{C} 353$, and 3C 405), was routinely observed every $1.5-2 \mathrm{hr}$ to correct for time-dependent variations in the gain of the instrument. There is a $5 \%$ uncertainty in the final flux density scale.

Further radio observations were obtained with the Australia Telescope Compact Array (ATCA; Frater, Brooks, \& Whiteoak 1992), at the Australia Telescope National Facility, on 1994 May 4-5, May 19-22, and August 30-31. PKS 2155-304 was monitored as part of a program to search for intraday variations in a sample of quasars and BL Lac objects. The ATCA consists of six $25 \mathrm{~m}$ antennas arranged in an east-west line, and observations were made at four wavelengths $(3,6,13$, and $20 \mathrm{~cm})$. Two slightly different configurations were used throughout the monitoring, $6 \mathrm{~A}$ and $6 \mathrm{D}$, with maximum baselines of 5939 and $5878 \mathrm{~m}$, respectively. The data were taken at 3 and $6 \mathrm{~cm}$ simultaneously, then at 13 and $20 \mathrm{~cm}$ after rotating the turrets. The correlator was configured in the standard way, with 32

TABLE 2

UMRAO RADIO DATA

\begin{tabular}{|c|c|c|c|c|}
\hline \multirow{2}{*}{$\begin{array}{c}\text { DATE OBSERVED } \\
(1994)\end{array}$} & \multirow{2}{*}{$\begin{array}{c}\text { JD } \\
(-2,440,000)\end{array}$} & \multicolumn{3}{|c|}{ FLux (mJy) } \\
\hline & & $4.8 \mathrm{GHz}$ & $8.0 \mathrm{GHz}$ & $14.5 \mathrm{GHz}$ \\
\hline Apr 29 & $9,471.9708$ & 470 & $\ldots$ & \\
\hline May $2 .$. & $9,475.0574$ & $\ldots$ & $\ldots$ & 450 \\
\hline May $3 .$. & $9,475.9784$ & $\ldots$ & & 420 \\
\hline May $4 \ldots .$. & $9,477.0253$ & $\ldots$ & 490 & \\
\hline May $5 \ldots \ldots \ldots \ldots$ & $9,478.0535$ & $\ldots$ & $\ldots$ & 430 \\
\hline May $6 \ldots \ldots \ldots \ldots$ & $9,478.9718$ & $\ldots$ & $\ldots$ & 440 \\
\hline May $10 \ldots \ldots \ldots$ & $9,482.9608$ & 380 & $\ldots$ & $\ldots$ \\
\hline May $19 \ldots \ldots \ldots$ & $9,491.9450$ & $\ldots$ & 420 & \\
\hline May $20 \ldots \ldots \ldots$ & $9,493.0255$ & .. & $\ldots$ & 520 \\
\hline May $27 \ldots \ldots \ldots$ & $9,499.9081$ & 490 & & $\ldots$ \\
\hline Jul $31 \ldots \ldots \ldots \ldots$ & $9,564.8854$ & $\ldots$ & 380 & $\ldots$ \\
\hline Sep 15 & $9,610.6574$ & $\ldots$ & & 510 \\
\hline Sep $17 \ldots \ldots \ldots \ldots$ & $9,612.7281$ & & 720 & $\ldots$ \\
\hline Sep $21 \ldots \ldots \ldots \ldots$ & $9,616.6107$ & 540 & & $\ldots$ \\
\hline $\operatorname{Dec} 4 \ldots \ldots \ldots \ldots$ & $9,690.5060$ & $\ldots$ & 520 & $\ldots$ \\
\hline
\end{tabular}

Notes.-Data from the University of Michigan Radio Astronomy Observatory (UMRAO) courtesy of M. F. A. and H. D. A. Typical uncertainties on individual measurements are 40,80 , and $20 \mathrm{mJy}$ at 4.8, 8.0, and $14.5 \mathrm{GHz}$, respectively. channels across a bandwidth of $128 \mathrm{MHz}$ for each wavelength. During the monitoring program the source was scanned four times every $24 \mathrm{hr}$, on average. Each scan lasted 1 minute with integration times of $10 \mathrm{~s}$. A turret was rotated between two pairs of wavelengths every second minute to provide almost simultaneous coverage of the available radio spectrum, with two orthogonal linear polarizations being measured.

The flux density scale was set on the standard primary calibrator used at ATCA, PKS 1934-638. The changes in phase caused by the receiver, local oscillator, and atmosphere were calibrated on the nearby point source (the secondary calibrator), PKS 2149-307. The flux densities given in Table 3 are the averages over all 13 baselines, with the exception of the two shortest baselines in order to reduce the influence of the extended structure of PKS 2155-304 and other confusing sources in the field. This is particularly important at $20 \mathrm{~cm}$, where the size of the primary beam is the largest $\left(33^{\prime}\right)$.

\subsection{Millimeter}

Observations were made using the $15 \mathrm{~m}$ Swedish-ESO Submillimetre Telescope (SEST), ${ }^{29}$ located on La Silla, Chile (Booth et al. 1989), with the SEST facility bolometer. PKS 2155-304 was observed on 1994 May 19 and 21 at 94 GHz and on 1994 April 24-25 and June 1, 25, and 26 at 90 and $230 \mathrm{GHz}$ (Table 4). Uranus was used as the primary flux calibrator and was checked by the secondary calibrator, Jupiter.

\subsection{Optical and Near-Infrared Photometry, Polarimetry, and Spectroscopy}

We arranged considerable observational coverage, but bad weather at several sites prevented the almost continuous level originally planned. The difficulty of obtaining continuous optical/near-infrared monitoring from the ground was exacerbated by the fact that the object was $\sim 90^{\circ}$ from the Sun in May, as required for the space-based observations. After May, weather and Sun-angle conditions

\footnotetext{
${ }^{29}$ The Swedish-ESO Submillimetre Telescope, SEST, is operated jointly by ESO and the Swedish National Facility for Radio Astronomy, Onsala Space Observatory at Chalmers University of Technology.
} 
TABLE 3

ATCA Centimeter Radio Data

\begin{tabular}{|c|c|c|c|c|c|}
\hline \multirow{2}{*}{$\begin{array}{c}\text { DATE OBSERVED } \\
(1994)\end{array}$} & \multirow{2}{*}{$\begin{array}{c}\text { JD } \\
(-2,440,000)\end{array}$} & \multicolumn{4}{|c|}{ FLUX (mJy) } \\
\hline & & $1.380 \mathrm{GHz}(20 \mathrm{~cm})$ & $2.378 \mathrm{GHz}(13 \mathrm{~cm})$ & $4.800 \mathrm{GHz}(6 \mathrm{~cm})$ & $8.640 \mathrm{GHz}(3 \mathrm{~cm})$ \\
\hline \multirow[t]{13}{*}{ May $4 \ldots \ldots \ldots \ldots$} & $9,477.1708$ & $378.1 \pm 48.9$ & $383.1 \pm 26.3$ & & \\
\hline & $9,477.2007$ & $\ldots$ & & $404.9 \pm 21.7$ & $336.5 \pm 19.9$ \\
\hline & $9,477.2236$ & $377.2 \pm 44.8$ & $380.0 \pm 24.7$ & & \\
\hline & $9,477.2576$ & & & $392.4 \pm 21.8$ & $326.5 \pm 18.7$ \\
\hline & $9,477.2806$ & $369.2 \pm 48.0$ & $374.1 \pm 23.4$ & & \\
\hline & $9,477.3160$ & & & $394.2 \pm 20.5$ & $328.7 \pm 17.9$ \\
\hline & $9,477.3368$ & $369.3 \pm 86.6$ & $376.0 \pm 22.8$ & & \\
\hline & $9,477.3931$ & & & $401.2 \pm 20.7$ & $331.5 \pm 18.3$ \\
\hline & $9,477.4063$ & $357.8 \pm 33.2$ & $377.7 \pm 24.1$ & & \\
\hline & $9,477.4236$ & & & $400.8 \pm 21.1$ & $330.0 \pm 18.2$ \\
\hline & $9,477.4465$ & $373.4 \pm 82.2$ & $384.1 \pm 22.9$ & & \\
\hline & $9,477.4688$ & $\ldots$ & & $401.7 \pm 21.8$ & $332.6 \pm 18.5$ \\
\hline & $9,477.4917$ & $376.2 \pm 64.1$ & $386.2 \pm 23.6$ & $\ldots$ & $\ldots$ \\
\hline \multirow{5}{*}{ May $5 \ldots \ldots \ldots . .}$. & $9,477.5271$ & $368.0 \pm 47.8$ & $390.0 \pm 24.7$ & & \\
\hline & $9,477.5493$ & & & $406.8 \pm 22.1$ & $332.1 \pm 20.0$ \\
\hline & $9,477.5722$ & $370.9 \pm 47.4$ & $392.5 \pm 26.5$ & & \\
\hline & $9,477.5951$ & & & $413.3 \pm 24.3$ & $337.7 \pm 21.8$ \\
\hline & $9,477.6097$ & $377.9 \pm 51.2$ & $392.7 \pm 29.7$ & & \\
\hline \multirow[t]{4}{*}{ May $19 \ldots \ldots \ldots$} & $9,492.1972$ & & & $417.5 \pm 30.2$ & $406.2 \pm 27.6$ \\
\hline & $9,492.1979$ & $395.1 \pm 35.0$ & $406.9 \pm 32.8$ & $\ldots$ & \\
\hline & $9,492.2764$ & & & $412.6 \pm 27.9$ & $399.4 \pm 26.9$ \\
\hline & $9,492.2778$ & $377.0 \pm 37.7$ & $400.4 \pm 31.6$ & & \\
\hline \multirow[t]{12}{*}{ May $20 \ldots \ldots \ldots$} & $9,492.5583$ & & & $422.4 \pm 33.4$ & $409.5 \pm 34.0$ \\
\hline & $9,492.5597$ & $378.1 \pm 40.4$ & $405.2 \pm 40.0$ & $\ldots$ & \\
\hline & $9,493.1368$ & & & $434.3 \pm 30.0$ & $414.5 \pm 33.2$ \\
\hline & $9,493.1375$ & $392.7 \pm 39.0$ & $417.0 \pm 35.2$ & $\ldots$ & $\ldots$ \\
\hline & $9,493.1958$ & & & $429.3 \pm 27.9$ & $415.5 \pm 29.2$ \\
\hline & $9,493.1965$ & $405.5 \pm 41.5$ & $422.5 \pm 32.5$ & $\ldots$ & .. \\
\hline & $9,493.2660$ & & & $426.4 \pm 27.8$ & $415.1 \pm 28.2$ \\
\hline & $9,493.2667$ & $373.6 \pm 37.5$ & $412.2 \pm 32.3$ & $\ldots$ & \\
\hline & $9,493.3708$ & & & $429.2 \pm 26.6$ & $419.7 \pm 28.5$ \\
\hline & $9,493.3715$ & $360.8 \pm 32.1$ & $404.1 \pm 32.0$ & & \\
\hline & $9,493.4583$ & & & $431.0 \pm 29.6$ & $427.8 \pm 29.5$ \\
\hline & $9,493.4597$ & $366.2 \pm 40.1$ & $404.8 \pm 33.8$ & $\ldots$ & \\
\hline \multirow{4}{*}{ May $21 \ldots . . . .}$. & $9,494.1958$ & & & $422.9 \pm 28.2$ & $421.8 \pm 28.8$ \\
\hline & $9,494.1965$ & $366.3 \pm 40.4$ & $392.1 \pm 33.1$ & $\ldots$ & $\ldots$ \\
\hline & $9,494.2708$ & & & $416.0 \pm 27.0$ & $416.3 \pm 28.4$ \\
\hline & $9,494.2722$ & $361.1 \pm 34.6$ & $390.7 \pm 30.6$ & & \\
\hline \multirow[t]{8}{*}{ May $22 \ldots . . . .}$. & $9,494.5611$ & & & $427.3 \pm 34.9$ & $421.5 \pm 35.7$ \\
\hline & $9,494.5618$ & $383.1 \pm 43.1$ & $395.0 \pm 43.3$ & & \\
\hline & $9,495.1243$ & & & $429.4 \pm 31.0$ & $434.7 \pm 34.0$ \\
\hline & $9,495.1250$ & $369.0 \pm 46.6$ & $384.2 \pm 36.4$ & $\ldots$ & $\ldots$ \\
\hline & $9,495.1826$ & & & $423.3 \pm 28.8$ & $433.8 \pm 29.7$ \\
\hline & $9,495.1840$ & $367.7 \pm 39.5$ & $387.3 \pm 34.8$ & $\ldots$ & $\ldots$ \\
\hline & $9,495.2528$ & & & $421.2 \pm 28.3$ & $428.1 \pm 30.2$ \\
\hline & $9,495.2542$ & $367.1 \pm 32.0$ & $381.9 \pm 32.5$ & $\ldots$ & $\ldots$ \\
\hline \multirow{10}{*}{ Aug $30 \ldots \ldots \ldots$} & $9,594.9340$ & $474.9 \pm 35.0$ & $467.8 \pm 32.6$ & & \\
\hline & $9,594.9354$ & & & $482.2 \pm 30.9$ & $479.7 \pm 35.9$ \\
\hline & $9,595.0035$ & $455.3 \pm 32.1$ & $463.8 \pm 32.7$ & & \\
\hline & $9,595.0049$ & & & $478.1 \pm 33.3$ & $477.1 \pm 48.7$ \\
\hline & $9,595.0889$ & $460.0 \pm 32.8$ & $461.7 \pm 32.9$ & & \\
\hline & $9,595.0903$ & & & $473.9 \pm 34.2$ & $465.4 \pm 45.3$ \\
\hline & $9,595.1785$ & $480.7 \pm 36.2$ & $466.6 \pm 34.1$ & & \\
\hline & $9,595.1799$ & & & $476.2 \pm 29.6$ & $472.2 \pm 34.9$ \\
\hline & $9,595.2653$ & $493.8 \pm 37.6$ & $474.7 \pm 36.1$ & $\ldots$ & \\
\hline & $9,595.2674$ & & & $491.4 \pm 30.6$ & $484.8 \pm 32.3$ \\
\hline \multirow[t]{10}{*}{ Aug $31 \ldots \ldots \ldots . .}$. & $9,595.8764$ & $508.6 \pm 36.8$ & $486.2 \pm 33.0$ & $\cdots$ & \\
\hline & $9,595.8778$ & & & $486.3 \pm 30.1$ & $484.8 \pm 30.0$ \\
\hline & $9,595.9813$ & $502.8 \pm 34.7$ & $475.2 \pm 31.1$ & & \\
\hline & $9,595.9826$ & & & $476.2 \pm 26.0$ & $471.5 \pm 27.0$ \\
\hline & $9,596.0639$ & $496.2 \pm 33.5$ & $476.9 \pm 32.1$ & $\ldots$ & \\
\hline & $9,596.0653$ & & & $481.5 \pm 25.9$ & $475.9 \pm 27.8$ \\
\hline & $9,596.1597$ & $497.4 \pm 32.5$ & $477.9 \pm 32.2$ & $\ldots$ & $\ldots$ \\
\hline & $9,596.1604$ & & & $483.2 \pm 28.0$ & $479.8 \pm 28.3$ \\
\hline & $9,596.2556$ & $503.0 \pm 43.3$ & $476.5 \pm 35.5$ & & \\
\hline & $9,596.2569$ & $\ldots$ & $\ldots$ & $487.7 \pm 30.2$ & $481.2 \pm 31.5$ \\
\hline
\end{tabular}

NotE.-Data from L. K.-C. 
TABLE 4

SEST Millimeter DATA

\begin{tabular}{|c|c|c|c|c|}
\hline \multirow{2}{*}{$\begin{array}{c}\text { DATE OBSERVED } \\
(1994)\end{array}$} & \multirow{2}{*}{$\begin{array}{c}\text { JD } \\
(-2,440,000)\end{array}$} & \multicolumn{3}{|c|}{ FLuX (mJy) } \\
\hline & & $90 \mathrm{GHz}^{\mathrm{a}}$ & $94 \mathrm{GHz}^{\mathrm{b}}$ & $230 \mathrm{GHz}^{\mathrm{a}}$ \\
\hline Apr 24 & $9,467.139$ & $355 \pm 71$ & $\ldots$ & $\cdots$ \\
\hline \multirow[t]{2}{*}{ Apr $25 \ldots \ldots \ldots$} & 9,468 & $348 \pm 41$ & .. & $\ldots$ \\
\hline & $9,468.215$ & $417 \pm 57$ & & $\ldots$ \\
\hline May 19 & $9,492.094$ & ... & $367 \pm 78$ & $\ldots$ \\
\hline May 21. & $9,494.104$ & & $367 \pm 78$ & • \\
\hline Jun $1 \ldots \ldots \ldots \ldots$ & $9,504.778$ & $430 \pm 81$ & $\ldots$ & $\ldots$ \\
\hline \multirow[t]{2}{*}{ Jun $25 \ldots \ldots \ldots$} & $9,528.751$ & $\ldots$ & $\ldots$ & $310 \pm 2$ \\
\hline & 9528.788 & & $\ldots$ & $330 \pm 25$ \\
\hline Jun 26 & $9,529.754$ & $450 \pm 117$ & $\ldots$ & $\ldots$ \\
\hline
\end{tabular}

${ }^{a}$ Data from M. T.

${ }^{b}$ Data from L. B. G. K.

improved, and monitoring observations continued to 1994 November.

Table 5 lists the 20 optical and near-infrared observers and telescopes that contributed to this campaign. Limited near-infrared data were available during the middle of the campaign and are given in Table 6. Exposure times were typically $\sim 40 \mathrm{~s}$ for $J H K$ and $\sim 40-160 \mathrm{~s}$ for $L$. Observations by $M . K$. were in the ESO IR system and have been converted to the South African Astronomical Observatory (SAAO) system following Carter (1990). For the optical data (Table 7), instrumental magnitudes were converted to $U B V R I$ magnitudes (Johnson $U B V$ and Cousins RI filters) using calibration stars in the field (Hamuy \& Maza 1989; Smith, Jannuzi, \& Elston 1991). Typical exposure times were $60-120 \mathrm{~s}(U), 20-600 \mathrm{~s}(B), 30-300 \mathrm{~s}(V), 20-300 \mathrm{~s}(R)$, and $30-120 \mathrm{~s}(I)$, and the errors are in the range $\lesssim 0.01$ to $\sim 0.08 \mathrm{mag}$, with $0.01 \mathrm{mag}$ being a typical value. Some of our observations were obtained at relatively high air mass, and the differential photometry does not adequately address the second-order extinction term. However, this term should account for no more than $0.03 \mathrm{mag}$.

Optical polarization measurements of PKS 2155-304 were made between 1994 May 13 and 21 (MJD 9,485-9,493) using the Two-Holer polarimeter/photometer (Table 8). The instrument, observational procedures, and data reduction are described in detail by Smith et al. (1992). An $8^{\prime \prime}$ circular aperture was used for all of the polarimetry, and typical exposure times were 3-8 minutes.

Optical polarimetry of PKS 2155-304 was also performed by the group at the University of São Paulo (USP) with their CCD imaging polarimeter (Table 9). The instrument was used at the Laboratório Nacional de Astrofisica (LNA), Brasópolis, with the LNA $1.60 \mathrm{~m}$ and USP $0.61 \mathrm{~m}$ telescopes, and is described in detail by Magalhães et al. (1996). Measurement errors are consistent with photon noise. Instrumental Stokes $Q, U$-values were converted to the equatorial system from standard-star data obtained on

TABLE 5

Optical/Near-INFrared ObSERVERS AND Telescopes

\begin{tabular}{|c|c|c|}
\hline Observer & Telescope & Filters \\
\hline A. J. B. & Lick $3 \mathrm{~m}$ & \\
\hline D. A. H. B. & AO $1.9 \mathrm{~m}$ & Johnson-Cousins $\left(U B V R_{C} I_{C}\right)$ \\
\hline E. C. ..... & ESO $1 \mathrm{~m}$ & Johnson-Cousins $\left(U B V R_{\mathrm{C}} I_{\mathrm{C}}\right)$ \\
\hline A. V. F.. & Lick $3 \mathrm{~m}$ & \\
\hline E. J. H. . & Steward 90 inch $(2.3 \mathrm{~m})$ & Johnson-Cousins $\left(B V R_{\mathrm{C}} I_{\mathrm{C}}\right)$ \\
\hline M. D. J. & CTIO $0.9 \mathrm{~m}$ & Johnson-Cousins $\left(B V R_{\mathrm{C}} I_{\mathrm{C}}\right)$ \\
\hline D. K. .... & SAAO $0.5 \mathrm{~m}$ & Johnson-Cousins $\left(U B V R_{\mathrm{C}} I_{\mathrm{C}}\right)$ \\
\hline M. K... & ESO $1 \mathrm{~m}$ & ESO $(J H K L)$ \\
\hline A. C. L.. & KPNO $0.9 \mathrm{~m}$ & Johnson-Cousins $\left(B V R_{\mathrm{C}} I_{\mathrm{C}}\right)$ \\
\hline A. C. L... & CTIO $0.9 \mathrm{~m}$ & Johnson-Cousins $\left(B V R_{\mathrm{C}} I_{\mathrm{C}}\right)$ \\
\hline A. M. M. . & Univ. São Paulo $0.61 \mathrm{~m}$ & Johnson $(B V)$ \\
\hline F. M. . & SAAO $0.5 \mathrm{~m}$ & Johnson-Cousins \\
\hline S. Ortolani & ESO $1.5 \mathrm{~m} \mathrm{D}$ & Johnson $(B V)$ \\
\hline J. E. P. & La Palma JK' & Johnson-Cousins $\left(B V R_{\mathrm{C}} I_{\mathrm{C}}\right)$ \\
\hline C. V. R. & Univ. São Paulo $0.61 \mathrm{~m}$ & Johnson $(B V)$ \\
\hline A. S. ..... & SAAO $1.9 \mathrm{~m}$ & SAAO $(J H K L)$ \\
\hline P. S. S. ... & Steward $1.5 \mathrm{~m}$ & Johnson-Cousins $\left(U B V R_{\mathrm{C}} I_{\mathrm{C}}\right)$ \\
\hline P.S. S. & Univ. Minnesota $1.5 \mathrm{~m}$ & Johnson-Cousins $\left(U B V R_{\mathrm{C}} I_{\mathrm{C}}\right)$ \\
\hline J. v. d. W. & & SAAO $(J H K L)$ \\
\hline F. v. W. & SAAO $0.5 \mathrm{~m}$ & Johnson-Cousins $\left(U B V R_{\mathrm{C}} I_{\mathrm{C}}\right)$ \\
\hline P. A. W. & SAAO $1.9 \mathrm{~m}$ & SAAO $(J H K L)$ \\
\hline S. J. W. & CTIO $0.9 \mathrm{~m}$ & Johnson-Cousins $\left(B V R_{\mathrm{C}} I_{\mathrm{C}}\right)$ \\
\hline
\end{tabular}

TABLE 6

NEAR-INFRARED DATA

\begin{tabular}{|c|c|c|c|c|c|c|}
\hline $\begin{array}{l}\text { Date Observed } \\
\text { (1994) }\end{array}$ & $\begin{array}{c}\text { JD } \\
(-2,440,000)\end{array}$ & $J$ & $H$ & K & $L$ & Observer \\
\hline May 19 & $9,491.68$ & $11.51 \pm 0.03$ & $10.82 \pm 0.03$ & $10.18 \pm 0.03$ & $9.14 \pm 0.05$ & P. A. W. \\
\hline May 20 & $9,492.66$ & $11.47 \pm 0.03$ & $10.78 \pm 0.03$ & $10.13 \pm 0.03$ & $9.06 \pm 0.05$ & P. A. W. \\
\hline \multirow[t]{2}{*}{ May $24 \ldots . . .}$. & $9,496.68$ & $11.36 \pm 0.03$ & $10.6 \pm 0.03$ & $9.98 \pm 0.03$ & $8.95 \pm 0.05$ & P. A. W. \\
\hline & $9,496.95$ & $11.51 \pm 0.02$ & $10.74 \pm 0.01$ & $9.99 \pm 0.02$ & $9.85 \pm 0.6$ & M. K. ${ }^{a}$ \\
\hline May 25 & $9,497.65$ & $11.34 \pm 0.03$ & $10.62 \pm 0.03$ & $9.99 \pm 0.03$ & $8.88 \pm 0.08$ & A. S./J. v. d. W. \\
\hline May $26 \ldots \ldots . .$. & $9,498.66$ & $11.30 \pm 0.03$ & $10.59 \pm 0.03$ & $9.93 \pm 0.03$ & $8.88 \pm 0.08$ & A. S./J. v. d. W. \\
\hline
\end{tabular}

a Magnitudes from M. K. were originally from the ESO standard system and have been converted to the SAAO system (see text). 
TABLE 7

Optical Data

\begin{tabular}{|c|c|c|c|c|c|c|c|}
\hline $\begin{array}{c}\text { Date Observed } \\
\text { (1994) }\end{array}$ & $\begin{array}{c}\text { JD } \\
(-2,440,000)\end{array}$ & $U$ & $B$ & $V$ & $R$ & $I$ & Observer \\
\hline May 2. & $9,474.9173$ & 12.33 & 13.21 & 13.02 & 12.76 & 12.31 & E. C. \\
\hline May $4 \ldots \ldots \ldots$ & $9,476.8737$ & 12.59 & 13.29 & 12.98 & 12.67 & 12.26 & E. C. \\
\hline May $5 \ldots \ldots \ldots$ & $9,477.8953$ & 12.65 & 13.29 & 13.03 & 12.73 & 12.33 & E. C. \\
\hline \multirow[t]{6}{*}{ May $8 \ldots . . . . .}$. & $9,480.9778$ & $\ldots$ & $\ldots$ & $\ldots$ & 12.83 & $\ldots$ & E. H. \\
\hline & $9,480.9813$ & $\ldots$ & 13.66 & $\ldots$ & $\ldots$ & $\ldots$ & E. H. \\
\hline & $9,480.9826$ & $\ldots$ & 13.67 & $\ldots$ & $\ldots$ & $\ldots$ & E. H. \\
\hline & $9,480.9861$ & $\ldots$ & $\ldots$ & 13.14 & $\ldots$ & $\ldots$ & E. H. \\
\hline & $9,480.9882$ & $\ldots$ & $\ldots$ & $\ldots$ & $\ldots$ & 12.42 & E. H. \\
\hline & $9,480.9896$ & & $\ldots$ & $\ldots$ & 12.84 & $\ldots$ & E. H. \\
\hline \multirow[t]{4}{*}{ May $11 \ldots . . .}$. & $9,483.6832$ & 13.17 & $\ldots$ & $\ldots$ & $\ldots$ & $\ldots$ & D. B. \\
\hline & $9,483.6867$ & $\ldots$ & $\ldots$ & 12.73 & $\ldots$ & $\ldots$ & D. B. \\
\hline & $9,483.6876$ & $\ldots$ & $\ldots$ & $\ldots$ & 12.46 & $\ldots$ & D. B. \\
\hline & $9,483.6884$ & & $\ldots$ & $\ldots$ & $\ldots$ & 12.46 & D. B. \\
\hline \multirow[t]{3}{*}{ May $12 \ldots \ldots .}$. & $9,484.6826$ & 13.13 & $\ldots$ & $\ldots$ & $\ldots$ & $\ldots$ & D. B. \\
\hline & $9,484.6857$ & $\ldots$ & $\ldots$ & 13.01 & . & $\ldots$ & D. B. \\
\hline & $9,484.6870$ & $\ldots$ & $\ldots$ & $\ldots$ & 12.66 & $\ldots$ & D. B. \\
\hline \multirow{5}{*}{ May $13 \ldots \ldots .}$. & $9,485.6762$ & 12.95 & $\ldots$ & $\ldots$ & $\ldots$ & $\ldots$ & D. B. \\
\hline & $9,485.6786$ & $\ldots$ & 13.28 & $\ldots$ & $\ldots$ & $\ldots$ & D. B. \\
\hline & $9,485.6794$ & $\ldots$ & $\ldots$ & 12.85 & $\ldots$ & $\ldots$ & D. B. \\
\hline & $9,485.6802$ & $\ldots$ & $\ldots$ & $\ldots$ & 12.67 & $\ldots$ & D. B. \\
\hline & $9,485.6810$ & $\ldots$ & $\ldots$ & $\ldots$ & $\ldots$ & 12.32 & D. B. \\
\hline \multirow[t]{9}{*}{ May $14 \ldots \ldots .}$. & $9,486.6609$ & 13.12 & $\ldots$ & $\ldots$ & $\ldots$ & $\ldots$ & D. B. \\
\hline & $9,486.6638$ & $\ldots$ & 13.35 & $\ldots$ & $\ldots$ & $\ldots$ & D. B. \\
\hline & $9,486.6645$ & $\ldots$ & $\ldots$ & 12.91 & . & $\ldots$ & D. B. \\
\hline & $9,486.6652$ & $\ldots$ & $\ldots$ & $\ldots$ & 12.66 & $\ldots$ & D. B. \\
\hline & $9,486.6659$ & $\ldots$ & $\ldots$ & $\ldots$ & $\ldots$ & 12.34 & D. B. \\
\hline & $9,486.6726$ & $\ldots$ & 13.22 & $\ldots$ & $\ldots$ & $\ldots$ & D. B. \\
\hline & $9,486.6734$ & $\ldots$ & $\ldots$ & 12.88 & $\ldots$ & $\ldots$ & D. B. \\
\hline & $9,486.6742$ & $\ldots$ & $\ldots$ & $\ldots$ & 12.58 & $\ldots$ & D. B. \\
\hline & $9,486.6753$ & $\ldots$ & $\ldots$ & $\ldots$ & $\ldots$ & 12.16 & D. B. \\
\hline \multirow{2}{*}{ May $15 \ldots \ldots .}$. & $9,487.6542$ & $\ldots$ & 13.21 & $\ldots$ & $\ldots$ & $\ldots$ & D. B. \\
\hline & $9,487.6575$ & $\ldots$ & & 12.79 & $\ldots$ & $\ldots$ & D. B. \\
\hline \multirow[t]{4}{*}{ May $16 \ldots . . .}$. & $9,488.6347$ & $\ldots$ & 13.15 & $\ldots$ & $\ldots$ & $\ldots$ & D. B. \\
\hline & $9,488.6382$ & $\ldots$ & $\ldots$ & 12.81 & & $\ldots$ & D. B. \\
\hline & $9,488.6595$ & $\ldots$ & $\ldots$ & $\ldots$ & 12.54 & $\ldots$ & D. B. \\
\hline & $9,488.6623$ & $\ldots$ & $\ldots$ & $\ldots$ & $\ldots$ & 12.11 & D. B. \\
\hline \multirow{2}{*}{ May $18 \ldots . . .}$. & $9,490.9888^{\mathrm{a}}$ & $\ldots$ & $\ldots$ & $\ldots$ & 12.55 & $\ldots$ & A. C. L. (KPNO) \\
\hline & $9,490.9919^{a}$ & $\ldots$ & $\ldots$ & 12.87 & $\ldots$ & $\ldots$ & A. C. L. (KPNO) \\
\hline \multirow{3}{*}{ May $19 \ldots . . .}$. & $9,491.9618^{\mathrm{a}}$ & $\ldots$ & $\ldots$ & 12.97 & $\ldots$ & $\ldots$ & P. S. S. \\
\hline & $9,491.9846^{\mathrm{a}}$ & $\ldots$ & $\ldots$ & & 12.64 & $\ldots$ & A. C. L. (KPNO) \\
\hline & $9,491.9869^{\mathrm{a}}$ & $\ldots$ & & 12.96 & $\ldots$ & $\ldots$ & A. C. L. (KPNO) \\
\hline May $20 \ldots \ldots .$. & $9,492.9271^{\mathrm{a}}$ & $\ldots$ & 13.20 & 12.81 & $\ldots$ & $\ldots$ & S. Ortolani \\
\hline & $9,492.9597^{a}$ & $\ldots$ & $\ldots$ & 12.82 & & $\ldots$ & P. S. S. \\
\hline & $9,492.9817^{\mathrm{a}}$ & $\ldots$ & $\ldots$ & $\ldots$ & 12.47 & $\ldots$ & A. C. L. (KPNO) \\
\hline May $21 \ldots . . .$. & $9,493.9563^{\mathrm{a}}$ & $\ldots$ & $\ldots$ & 12.69 & $\ldots$ & $\ldots$ & P. S. S. \\
\hline May $22 \ldots \ldots .$. & $9,494.7889^{a}$ & $\ldots$ & 13.20 & . & $\ldots$ & $\ldots$ & M. J. \\
\hline & $9,494.7917^{\mathrm{a}}$ & $\ldots$ & $\ldots$ & 12.86 & $\ldots$ & $\cdots$ & M. J. \\
\hline & $9,494.7937^{\mathrm{a}}$ & $\ldots$ & $\ldots$ & $\ldots$ & 12.62 & $\ldots$ & M. J. \\
\hline & $9,494.7954^{\mathrm{a}}$ & $\ldots$ & $\ldots$ & $\ldots$ & $\ldots$ & 12.18 & M. J. \\
\hline & $9,494.8664^{\mathrm{a}}$ & $\ldots$ & $\ldots$ & 12.90 & $\ldots$ & $\ldots$ & M. J. \\
\hline & $9,494.8693^{\mathrm{a}}$ & $\ldots$ & 13.24 & $\ldots$ & $\ldots$ & $\ldots$ & M. J. \\
\hline May $25 \ldots . . .$. & $9,497.8342^{\mathrm{a}}$ & $\ldots$ & $\ldots$ & $\ldots$ & $\ldots$ & 12.04 & M. J. \\
\hline & $9,497.8354^{\mathrm{a}}$ & $\ldots$ & $\ldots$ & $\ldots$ & $\ldots$ & 12.05 & M. J. \\
\hline & $9,497.8373^{\mathrm{a}}$ & $\ldots$ & $\ldots$ & $\ldots$ & 12.46 & $\ldots$ & M. J. \\
\hline & $9,497.8385^{\mathrm{a}}$ & $\ldots$ & $\ldots$ & $\ldots$ & 12.47 & $\ldots$ & M. J. \\
\hline & $9,497.8402^{\mathrm{a}}$ & $\ldots$ & $\ldots$ & 12.76 & $\ldots$ & $\ldots$ & M. J. \\
\hline & $9,497.8418^{a}$ & $\ldots$ & $\ldots$ & 12.81 & $\ldots$ & $\ldots$ & M. J. \\
\hline & $9,497.8454^{\mathrm{a}}$ & $\ldots$ & 13.06 & $\ldots$ & $\ldots$ & $\ldots$ & M. J. \\
\hline & $9,497.8491^{\mathrm{a}}$ & $\ldots$ & 13.06 & $\ldots$ & $\ldots$ & $\ldots$ & M. J. \\
\hline & $9,497.8528^{a}$ & $\ldots$ & 13.06 & $\ldots$ & $\ldots$ & $\ldots$ & M. J. \\
\hline May $26 \ldots . .$. & $9,498.8535^{\mathrm{a}}$ & $\ldots$ & $\ldots$ & 12.73 & $\ldots$ & $\ldots$ & A. M. M./C. V. R. \\
\hline & $9,498.8603^{\mathrm{a}}$ & $\ldots$ & 13.23 & $\ldots$ & $\ldots$ & $\ldots$ & A. M. M./C. V. R. \\
\hline & $9,498.8728^{a}$ & $\ldots$ & 13.08 & $\ldots$ & $\ldots$ & $\ldots$ & M. J. \\
\hline & $9,498.8750^{\mathrm{a}}$ & $\ldots$ & 13.08 & $\ldots$ & $\ldots$ & $\ldots$ & M. J. \\
\hline & $9,498.8776^{a}$ & $\ldots$ & $\ldots$ & 12.76 & $\ldots$ & $\ldots$ & M. J. \\
\hline & $9,498.8789^{a}$ & $\ldots$ & $\ldots$ & 12.76 & $\ldots$ & $\ldots$ & M. J. \\
\hline & $9,498.8805^{\mathrm{a}}$ & $\ldots$ & $\ldots$ & $\ldots$ & 12.45 & $\ldots$ & M. J. \\
\hline & $9,498.8814^{\mathrm{a}}$ & $\ldots$ & $\ldots$ & $\ldots$ & 12.44 & $\ldots$ & M. J. \\
\hline & $9,498.8832^{\mathrm{a}}$ & $\ldots$ & $\ldots$ & $\ldots$ & $\ldots$ & 12.04 & M. J. \\
\hline & $9,498.8841^{\mathrm{a}}$ & & $\ldots$ & $\ldots$ & $\ldots$ & 12.02 & M. J. \\
\hline May $27 \ldots \ldots .$. & $9,499.9454$ & $\ldots$ & $\ldots$ & 12.86 & $\ldots$ & $\ldots$ & S. J. W. \\
\hline
\end{tabular}


TABLE 7-Continued

\begin{tabular}{|c|c|c|c|c|c|c|c|}
\hline $\begin{array}{l}\text { Date Observed } \\
\text { (1994) }\end{array}$ & $\begin{array}{c}\text { JD } \\
(-2,440,000)\end{array}$ & $U$ & $B$ & $V$ & $R$ & $I$ & Observer \\
\hline & $9,499.9478$ & .. & & & 12.50 & & S. J. W. \\
\hline & $9,499.9495$ & $\ldots$ & $\ldots$ & & $\ldots$ & 12.14 & S. J. W. \\
\hline \multirow[t]{2}{*}{ May $31 \ldots . . .}$. & $9,503.9392$ & $\ldots$ & $\ldots$ & 12.74 & & $\ldots$ & S. J. W. \\
\hline & $9,503.9413$ & $\ldots$ & & $\ldots$ & 12.41 & $\ldots$ & S. J. W. \\
\hline \multirow[t]{3}{*}{ Jun $3 \ldots \ldots \ldots$} & $9,506.9386$ & $\ldots$ & 12.85 & & $\ldots$ & $\ldots$ & S. J. W. \\
\hline & $9,506.9411$ & $\ldots$ & $\ldots$ & 12.65 & $\ldots$ & . & S. J. W. \\
\hline & $9,506.9459$ & $\ldots$ & & $\ldots$ & $\ldots$ & 11.92 & S. J. W. \\
\hline \multirow{2}{*}{ Jun $4 \ldots \ldots \ldots \ldots$} & $9,507.9301$ & $\ldots$ & 12.94 & $\ldots$ & $\ldots$ & $\ldots$ & S. J. W. \\
\hline & $9,507.9325$ & & & 12.72 & & & S. J. W. \\
\hline Jun 12 & $9,515.5978$ & 12.15 & 12.85 & 12.55 & 12.25 & 11.86 & D. K./F. M./F. v. W. \\
\hline Jun 14 & $9,517.6211$ & 12.24 & 12.94 & 12.63 & 12.32 & 11.92 & D. K./F. M./F. v. W. \\
\hline Jun 15 . & $9,518.6302$ & 12.32 & 13.01 & 12.68 & 12.38 & 11.98 & D. K./F. M./F. v. W. \\
\hline Jun $16 \ldots \ldots \ldots$ & $9,519.6477$ & 12.38 & 13.07 & 12.74 & 12.44 & 12.08 & D. K./F. M./F. v. W. \\
\hline Jun $22 \ldots$ & $9,525.6015$ & 12.45 & 13.11 & 12.88 & 12.58 & 12.17 & D. K./F. M./F. v. W. \\
\hline \multirow[t]{3}{*}{ Jun $26 \ldots \ldots \ldots$} & $9,529.9427$ & $\ldots$ & $\ldots$ & & $\ldots$ & 12.15 & A. C. L. (CTIO) \\
\hline & $9,529.9438$ & $\ldots$ & & 12.86 & $\cdots$ & $\ldots$ & A. C. L. (CTIO) \\
\hline & $9,529.9452$ & $\ldots$ & 13.10 & $\ldots$ & $\cdots$ & $\begin{array}{l}\cdots \\
\cdots\end{array}$ & A. C. L. (CTIO) \\
\hline \multirow[t]{4}{*}{ Jun $27 \ldots \ldots \ldots$} & $9,530.8505$ & $\ldots$ & 13.06 & . & $\ldots$ & $\ldots$ & A. C. L. (CTIO) \\
\hline & $9,530.8538$ & $\ldots$ & $\ldots$ & 12.80 & $\ldots$ & & A. C. L. (CTIO) \\
\hline & $9,530.8554$ & $\ldots$ & $\ldots$ & $\ldots$ & $\ldots$ & 12.04 & A. C. L. (CTIO) \\
\hline & $9,530.8616$ & & & & & 12.10 & A. C. L. (CTIO) \\
\hline Jul 1 . & $9,534.6218$ & 12.31 & 13.02 & 12.71 & 12.42 & 12.03 & D. K./F. M./F. v. W. \\
\hline Jul 2 . & $9,535.6036$ & 12.36 & 13.05 & 12.73 & 12.43 & 12.03 & D. K./F. M./F. v. W. \\
\hline Jul 3 . & $9,536.6211$ & 12.35 & 13.05 & 12.74 & 12.44 & 12.06 & D. K./F. M./F. v. W. \\
\hline Jul 4 . & $9,537.5886$ & 12.26 & 12.97 & 12.68 & 12.37 & 12.00 & D. K./F. M./F. v. W. \\
\hline Jul $5 \ldots$ & $9,538.5756$ & 12.45 & 13.14 & 12.83 & 12.51 & 12.10 & D. K./F. M./F. v. W. \\
\hline Jul $6 \ldots$ & $9,539.5865$ & 12.45 & 13.15 & 12.82 & 12.52 & 12.12 & D. K./F. M./F. v. W. \\
\hline Jul 7 . & $9,540.5710$ & 12.44 & 13.14 & 12.82 & 12.52 & 12.11 & D. K./F. M./F. v. W. \\
\hline Jul 8 . & $9,541.5812$ & 12.43 & 13.12 & 12.80 & 12.49 & 12.07 & D. K./F. M./F. v. W. \\
\hline Jul 9 . & $9,542.6156$ & 12.39 & 13.10 & 12.79 & 12.49 & 12.10 & D. K./F. M./F. v. W. \\
\hline Jul 12 . & $9,545.5740$ & 12.34 & 13.04 & 12.74 & 12.44 & 12.04 & D. K./F. M./F. v. W. \\
\hline Jul 13 . & $9,546.5510$ & 12.38 & 13.09 & 12.78 & 12.48 & 12.08 & D. K./F. M./F. v. W. \\
\hline Jul 14 . & $9,547.5769$ & 12.41 & 13.10 & 12.79 & 12.49 & 12.10 & D. K./F. M./F. v. W. \\
\hline \multirow[t]{9}{*}{ Jul $20 \ldots \ldots \ldots$} & $9,553.6226$ & $\ldots$ & 13.02 & $\ldots$ & $\ldots$ & $\ldots$ & J. E. P. (JKT) \\
\hline & $9,553.6290$ & $\ldots$ & 13.02 & $\ldots$ & $\cdots$ & $\cdots$ & J. E. P. (JKT) \\
\hline & $9,553.6341$ & $\ldots$ & 13.02 & $\ldots$ & $\ldots$ & $\ldots$ & J. E. P. (JKT) \\
\hline & $9,553.6390$ & $\ldots$ & $\ldots$ & 12.67 & $\ldots$ & $\ldots$ & J. E. P. (JKT) \\
\hline & $9,553.6437$ & $\ldots$ & $\ldots$ & 12.66 & $\cdots$ & $\cdots$ & J. E. P. (JKT) \\
\hline & $9,553.6520$ & $\ldots$ & $\ldots$ & $\ldots$ & 12.35 & $\ldots$ & J. E. P. (JKT) \\
\hline & $9,553.6555$ & $\ldots$ & $\ldots$ & $\ldots$ & 12.34 & & J. E. P. (JKT) \\
\hline & $9,553.6618$ & $\ldots$ & $\ldots$ & $\ldots$ & $\ldots$ & 11.93 & J. E. P. (JKT) \\
\hline & $9,553.6682$ & & & & & 11.94 & J. E. P. (JKT) \\
\hline Jul 28 . & $9,561.5073$ & 12.14 & 12.84 & 12.52 & 12.22 & 11.80 & D. K./F. M./F. v. W. \\
\hline Jul 29 . & $9,562.5073$ & 12.13 & 12.82 & 12.53 & 12.23 & 11.83 & D. K./F. M./F. v. W. \\
\hline Jul 30 . & $9,563.5061$ & 12.22 & 12.92 & 12.62 & 12.31 & 11.91 & D. K./F. M./F. v. W. \\
\hline Aug 1 . & $9,566.4992$ & 12.19 & 12.89 & 12.58 & 12.28 & 11.91 & D. K./F. M./F. v. W. \\
\hline \multirow{8}{*}{ Aug $2 \ldots \ldots \ldots$} & $9,566.5353$ & $\ldots$ & 12.93 & 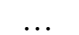 & . & $\ldots$ & J. E. P. (JKT) \\
\hline & $9,566.5425$ & $\ldots$ & 12.96 & & $\ldots$ & $\ldots$ & J. E. P. (JKT) \\
\hline & $9,566.5491$ & $\ldots$ & $\ldots$ & 12.60 & $\ldots$ & $\ldots$ & J. E. P. (JKT) \\
\hline & $9,566.5535$ & $\ldots$ & $\ldots$ & 12.62 & & $\ldots$ & J. E. P. (JKT) \\
\hline & $9,566.5561$ & $\ldots$ & $\ldots$ & $\ldots$ & 12.30 & $\ldots$ & J. E. P. (JKT) \\
\hline & $9,566.5604$ & $\ldots$ & $\ldots$ & $\ldots$ & 12.29 & & J. E. P. (JKT) \\
\hline & $9,566.5627$ & $\ldots$ & $\ldots$ & $\ldots$ & $\ldots$ & 11.90 & J. E. P. (JKT) \\
\hline & $9,566.5668$ & $\ldots$ & $\ldots$ & $\ldots$ & $\ldots$ & 11.89 & J. E. P. (JKT) \\
\hline \multirow[t]{2}{*}{ Aug $10 \ldots \ldots \ldots$} & $9,575.4039$ & 12.01 & 12.72 & 12.44 & 12.15 & 11.78 & D. K./F. M./F. v. W. \\
\hline & $9,575.4813$ & 12.00 & 12.71 & 12.42 & 12.13 & 11.75 & D. K./F. M./F. v. W. \\
\hline Aug $11 \ldots \ldots \ldots$ & $9,576.4048$ & 12.12 & 12.82 & 12.51 & 12.22 & 11.82 & D. K./F. M./F. v. W. \\
\hline & $9,576.4830$ & 12.12 & 12.82 & 12.51 & 12.21 & 11.82 & D. K./F. M./F. v. W. \\
\hline Aug 12 . & $9,576.5704$ & 12.12 & 12.82 & 12.51 & 12.22 & 11.81 & D. K./F. M./F. v. W. \\
\hline Aug $15 .$. & $9,580.4852$ & 11.96 & 12.65 & 12.36 & 12.07 & 11.68 & D. K./F. M./F. v. W. \\
\hline Aug 16 . & $9,580.5576$ & 11.97 & 12.67 & 12.36 & 12.07 & 11.66 & D. K./F. M./F. v. W. \\
\hline & $9,581.4061$ & 11.92 & 12.62 & 12.32 & 12.04 & 11.67 & D. K./F. M./F. v. W. \\
\hline & $9,581.4547$ & 11.90 & 12.60 & 12.31 & 12.01 & 11.63 & D. K./F. M./F. v. W. \\
\hline Aug $17 \ldots \ldots \ldots$ & $9,581.5542$ & 11.88 & 12.55 & 12.28 & 12.00 & 11.66 & D. K./F. M./F. v. W. \\
\hline & $9,582.3906$ & 11.93 & 12.61 & 12.32 & 12.02 & 11.62 & D. K./F. M./F. v. W. \\
\hline & $9,582.4599$ & 11.90 & 12.61 & 12.30 & 12.02 & 11.62 & D. K./F. M./F. v. W. \\
\hline Aug $18 .$. & $9,582.5257$ & 11.90 & 12.61 & 12.31 & 12.02 & 11.65 & D. K./F. M./F. v. W. \\
\hline Aug $21 .$. & $9,586.3861$ & 12.06 & 12.70 & 12.40 & 12.14 & 11.72 & D. K./F. M./F. v. W. \\
\hline & $9,586.4330$ & 12.04 & 12.76 & 12.44 & 12.13 & 11.75 & D. K./F. M./F. v. W. \\
\hline Aug $22 .$. & $9,586.5211$ & 12.07 & 12.81 & 12.49 & 12.16 & 11.79 & D. K./F. M./F. v. W. \\
\hline & $9,587.3876$ & 11.99 & 12.72 & 12.39 & 12.09 & 11.66 & D. K./F. M./F. v. W. \\
\hline & $9,587.4385$ & 11.99 & 12.73 & 12.42 & 12.09 & 11.69 & D. K./F. M./F. v. W. \\
\hline Aug $23 \ldots \ldots \ldots$ & $9,587.5223$ & 12.03 & 12.76 & 12.46 & 12.09 & 11.77 & D. K./F. M./F. v. W. \\
\hline
\end{tabular}


TABLE 7-Continued

\begin{tabular}{|c|c|c|c|c|c|c|c|}
\hline $\begin{array}{c}\text { Date Observed } \\
\text { (1994) }\end{array}$ & $\begin{array}{c}\text { JD } \\
(-2,440,000)\end{array}$ & $U$ & $B$ & $V$ & $R$ & $I$ & Observer \\
\hline Aug $25 .$. & $9,590.4331$ & 11.99 & 12.67 & 12.34 & 12.02 & 11.61 & D. K./F. M./F. v. W. \\
\hline Aug $29 \ldots \ldots \ldots$ & $9,594.4065$ & 11.98 & 12.68 & 12.36 & 12.04 & 11.65 & D. K./F. M./F. v. W. \\
\hline \multirow[t]{2}{*}{ Sep $2 \ldots \ldots \ldots \ldots$} & $9,597.5145$ & 11.91 & 12.61 & 12.31 & 12.01 & 11.59 & D. K./F. M./F. v. W. \\
\hline & $9,598.4908$ & 11.99 & 12.67 & 12.36 & 12.06 & 11.65 & D. K./F. M./F. v. W. \\
\hline Sep $5 .$. & $9,601.4694$ & 12.03 & 12.71 & 12.36 & 12.05 & 11.64 & D. K./F. M./F. v. W. \\
\hline Sep $8 \ldots$ & $9,604.3906$ & 11.90 & 12.59 & 12.27 & 11.96 & 11.55 & D. K./F. M./F. v. W. \\
\hline Sep 11 . & $9,607.3721$ & 12.00 & 12.70 & 12.39 & 12.09 & 11.71 & D. K./F. M./F. v. W. \\
\hline Sep 12 . & $9,608.3965$ & 11.87 & 12.57 & 12.28 & 11.98 & 11.59 & D. K./F. M./F. v. W. \\
\hline \multirow[t]{4}{*}{ Nov $16 \ldots \ldots \ldots$} & $9,672.6097$ & $\ldots$ & & $\ldots$ & 12.44 & $\ldots$ & E. H. \\
\hline & $9,672.6167$ & $\ldots$ & 13.24 & & $\ldots$ & $\ldots$ & E. H. \\
\hline & $9,672.6285$ & $\ldots$ & $\ldots$ & 12.74 & $\ldots$ & $\ldots$ & E. H. \\
\hline & $9,672.6403$ & $\ldots$ & $\ldots$ & $\ldots$ & $\ldots$ & 12.01 & E. H. \\
\hline
\end{tabular}

NoTE.-For all passbands, uncertainties are typically $0.01 \mathrm{mag}$.

a Simultaneous space-based data available.

the same night. The instrumental polarization was measured to be less than $0.03 \%$; being considerably smaller than the measured errors, no correction has been applied to the data.

TABLE 8

Two-Holer Polarization Data

\begin{tabular}{|c|c|c|c|c|}
\hline $\begin{array}{c}\text { Date Observed } \\
\text { (1994) }\end{array}$ & $\begin{array}{c}\text { JD } \\
(-2,440,000)\end{array}$ & Filter & $\begin{array}{c}P \\
(\%)\end{array}$ & $\begin{array}{l}\text { P.A. } \\
\text { (deg) }\end{array}$ \\
\hline May 13 & $9,485.97$ & V & $7.57 \pm 0.67$ & $96.5 \pm 2.5$ \\
\hline May 14 & $9,486.96$ & $V$ & $7.52 \pm 0.81$ & $101.7 \pm 3.1$ \\
\hline May $15 \ldots$ & $9,487.96$ & $V$ & $2.88 \pm 0.29$ & $98.0 \pm 2.9$ \\
\hline May $16 .$. & $9,488.96$ & $V$ & $4.99 \pm 0.22$ & $108.9 \pm 1.3$ \\
\hline May $17 \ldots$ & $9,489.95$ & $V$ & $6.54 \pm 0.36$ & $132.1 \pm 1.6$ \\
\hline \multirow[t]{5}{*}{ May $19 \ldots \ldots .}$. & $9,491.95$ & $U$ & $5.53 \pm 0.94$ & $157.1 \pm 4.8$ \\
\hline & $9,491.96$ & $B$ & $6.23 \pm 0.37$ & $151.1 \pm 1.7$ \\
\hline & $9,491.96$ & $V$ & $5.92 \pm 0.28$ & $150.1 \pm 1.3$ \\
\hline & $9,491.95$ & $R$ & $5.68 \pm 0.29$ & $151.2 \pm 1.4$ \\
\hline & $9,491.95$ & $I$ & $5.38 \pm 0.30$ & $150.8 \pm 1.6$ \\
\hline \multirow[t]{5}{*}{ May $20 \ldots \ldots \ldots$} & $9,492.95$ & $U$ & $8.84 \pm 0.48$ & $137.9 \pm 1.6$ \\
\hline & $9,492.94$ & $B$ & $8.76 \pm 0.35$ & $136.4 \pm 1.1$ \\
\hline & $9,492.96$ & $V$ & $8.02 \pm 0.30$ & $135.3 \pm 1.1$ \\
\hline & $9,492.94$ & $R$ & $7.54 \pm 0.33$ & $134.9 \pm 1.2$ \\
\hline & $9,492.95$ & $I$ & $7.70 \pm 0.36$ & $135.5 \pm 1.3$ \\
\hline \multirow[t]{5}{*}{ May $21 \ldots . . .}$. & $9,493.95$ & $U$ & $14.25 \pm 0.35$ & $133.0 \pm 0.7$ \\
\hline & $9,493.94$ & $B$ & $13.19 \pm 0.30$ & $131.8 \pm 0.6$ \\
\hline & $9,493.95$ & $V$ & $12.31 \pm 0.21$ & $131.5 \pm 0.5$ \\
\hline & $9,493.93$ & $R$ & $11.90 \pm 0.21$ & $132.0 \pm 0.5$ \\
\hline & $9,493.94$ & $I$ & $10.89 \pm 0.24$ & $132.6 \pm 0.6$ \\
\hline
\end{tabular}

NoTE.-Observations by P. S. S. with the University of Minnesota $1.5 \mathrm{~m}$ (1994 May 13-17) and the Steward Observatory $1.5 \mathrm{~m}$ (1994 May 19-21) telescopes, both on Mount Lemmon, Arizona.

TABLE 9

LNA AND USP POLARIZATION DATA

\begin{tabular}{|c|c|c|c|c|}
\hline $\begin{array}{c}\text { Date Observed } \\
\text { (1994) }\end{array}$ & $\begin{array}{c}\text { JD } \\
(-2,440,000)\end{array}$ & Filter & $\begin{array}{c}P \\
(\%)\end{array}$ & $\begin{array}{l}\text { P.A. } \\
\text { (deg) }\end{array}$ \\
\hline \multirow[t]{2}{*}{ Jul $2 \ldots \ldots \ldots \ldots$} & $9,535.80$ & V & $11.29 \pm 0.15$ & $105.0 \pm 0.4$ \\
\hline & 9,535 & $R$ & $10.28 \pm 0.05$ & 101.6 \\
\hline Jul 3 & $9,536.78$ & None & $10.22 \pm 0.08$ & $104.3 \pm 0.2$ \\
\hline \multirow[t]{2}{*}{ Jul $5 \ldots \ldots \ldots$} & $9,538.83$ & B & $10.04 \pm 0.14$ & $114.1 \pm 0.4$ \\
\hline & $9,538.71$ & $I$ & $9.20 \pm 0.09$ & $118.9 \pm 0.3$ \\
\hline \multirow[t]{2}{*}{ Jul $21 \ldots \ldots \ldots$} & $9,554.83$ & $V$ & $8.12 \pm 0.15$ & $94.7 \pm 0.5$ \\
\hline & $9,554.79$ & $R$ & $7.33 \pm 0.10$ & $94.1 \pm 0.4$ \\
\hline Jul 25 & $9,558.70$ & $I$ & $7.38 \pm 0.07$ & $101.4 \pm 0.3$ \\
\hline Sep 1. & $9,596.79$ & V & $5.36 \pm 0.04$ & $95.1 \pm 0.2$ \\
\hline \multirow[t]{2}{*}{ Oct $13 \ldots \ldots \ldots$} & $9,638.53$ & $B$ & $5.16 \pm 0.06$ & $96.8 \pm 0.3$ \\
\hline & $9,638.57$ & V & $5.36 \pm 0.07$ & $95.5 \pm 0.4$ \\
\hline
\end{tabular}

Note.-Observations by A. M. M., V. E. M., A. P., and C. V. R. with the LNA $1.60 \mathrm{~m}$ and USP $0.61 \mathrm{~m}$ telescopes.
Optical spectra of PKS 2155-304 were obtained in morning twilight on 1994 June 3 (MJD 9,506.9875) with the Kast double spectrograph (Miller \& Stone 1993) at the Cassegrain focus of the Shane $3 \mathrm{~m}$ reflector at Lick Observatory. Reticon $400 \times 1200$ pixel CCDs were used in both cameras. A long slit of width $4^{\prime \prime}$ was oriented along the parallactic angle to minimize differential light losses produced by atmospheric dispersion. Several different grating and grism settings were required to cover the entire accessible wavelength range (3220-9908 $\AA$ ), with a resolution of 8-11 $\AA$. The standard stars BD $+26^{\circ} 2606$ (Oke \& Gunn 1983) and Feige 34 (Massey et al. 1988) were used for flux calibration. These were also used to eliminate (through division) the telluric absorption bands in the spectrum of PKS 2155-304. The atmospheric seeing during the observations was poor and variable $\left(\sim 3^{\prime \prime}-4^{\prime \prime}\right)$. Moreover, the extinction correction cannot be fully trusted because the air mass was high (3.0-3.2). Thus, although the night was clear, the derived absolute flux for the final spectrum might be somewhat erroneous. The relative flux calibration, on the other hand, should be more reliable, except perhaps at the near-ultraviolet wavelengths.

\section{RESULTS AND DISCUSSION}

\subsection{Radio Results}

The radio fluxes show evidence of variability at the level of a few percent at all frequencies (see Fig. 1) and are better sampled than during the previous campaign in 1991 November, when the flux increased by $20 \%$ in 1 month (Courvoisier et al. 1995). The trend in the high-frequency data $(22.5,15$, and $8.4 \mathrm{GHz})$ is an increase of about $10 \%$ from May 14 until around May 24 (MJD 9,487-9,497), when the flux begins to decline. This trend may also be present in the less well sampled $5 \mathrm{GHz}$ data, though probably not at $1.5 \mathrm{GHz}$. The variability amplitude appears to increase with increasing frequency, from $\sim 10 \%$ to $\sim 20 \%$ for the 8.4 $22.5 \mathrm{GHz}$ data, and the peak at 8.4 and $15 \mathrm{GHz}$ appears to occur simultaneously, while the $22.5 \mathrm{GHz}$ peak seems to have occurred about 5 days earlier. There is no discernible change in the radio spectral index, unlike the case in the previous campaign, in which the radio spectrum flattened over the period of observation (Courvoisier et al. 1995). The large $22.5 \mathrm{GHz}$ peak on May 26 (MJD 9,499.05) is probably an artifact due to calibration uncertainties. The Michigan data do not show the same behavior because of the lack of coverage. 


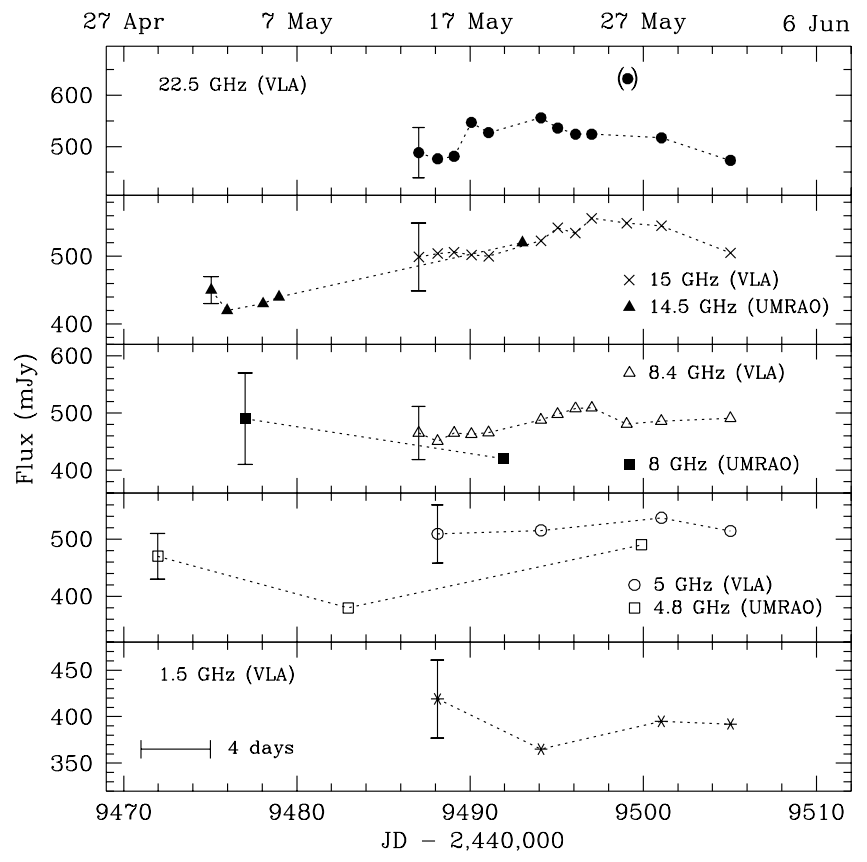

FIG. 1.-Radio light curves from 1994 April to June. The 22.5, 15, and $8.4 \mathrm{GHz}$ data show a slight $(10 \%-20 \%)$ increase in flux and then a decrease by the same amount over the observation period. The $14.5 \mathrm{GHz}$ data increase by the same amount, while the other bands are basically invariant. Uncertainties are shown on the first point of each light curve and are $\pm 10 \%$ for the VLA data and $\pm 40, \pm 80$, and $\pm 20 \mathrm{mJy}$ for the UMRAO $4.8,8.0$, and $14.5 \mathrm{GHz}$ data, respectively. The large $22.5 \mathrm{GHz}$ peak on May 26 (MJD 9,499.05) is probably an artifact. The dotted lines have been added to guide the eye only.

The lower frequency radio data (ATCA) show no variability over the three periods of observation. Variations of $20 \%$ would have been observed easily but are not seen (Fig. 2). PKS $2155-304$ does increase in brightness by $20 \%-$ $40 \%$ from early May to late August at all four wavelengths. Although by a smaller factor, this corresponds to the general brightening of the source in the optical over the same period (see below). A marked change in spectral index is observed for these data, with the spectrum inverting from early to mid May and flattening by the last observations in late August. During the SEST observations the source

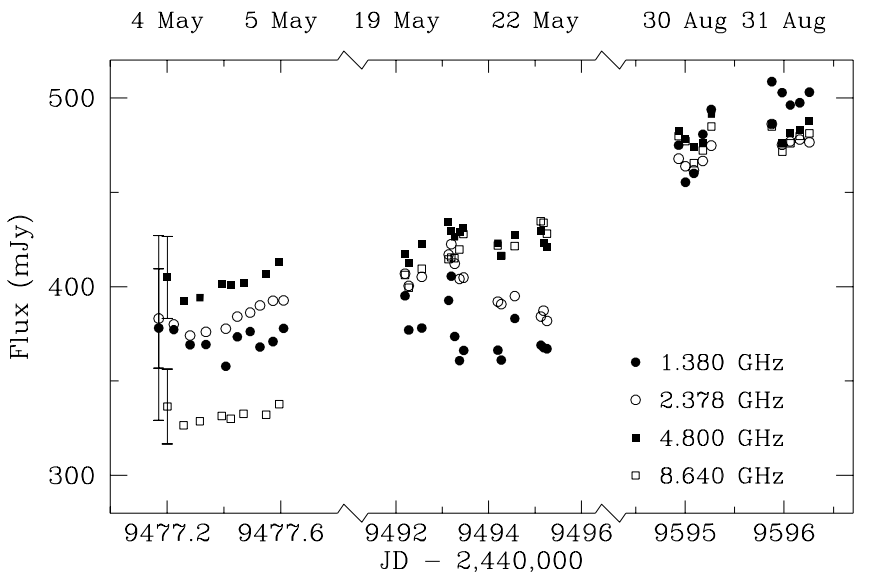

Fig. 2. - Centimeter light curves from ATCA. The source brightens slightly $(20 \%-40 \%)$ over the observation period (May-August), and there is a strong change in the spectral index between early and mid May and again between mid May and late August.

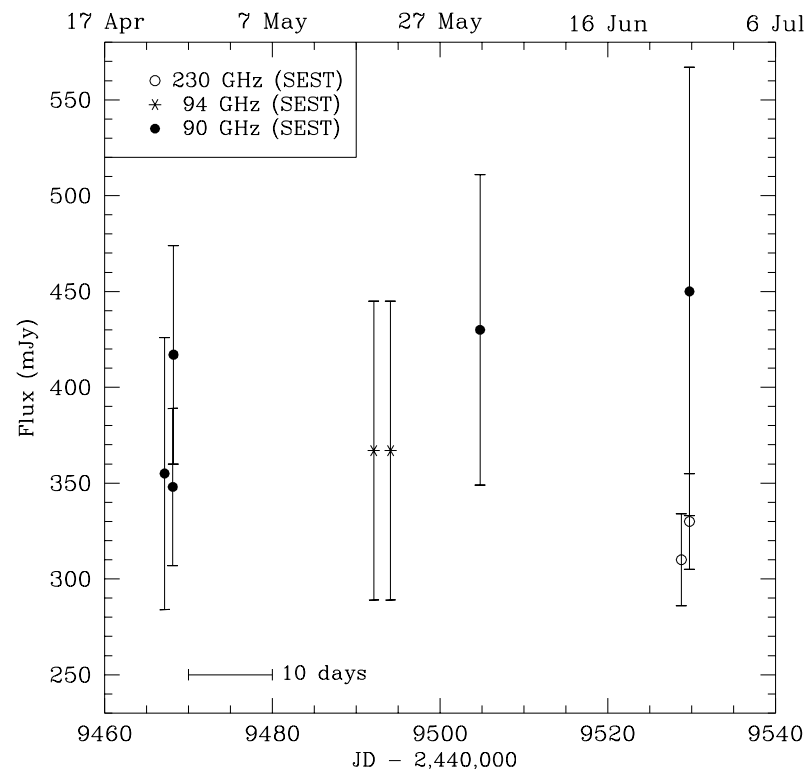

FIG. 3.-Millimeter light curves from SEST. No variations are obvious. A timescale bar is shown for comparison with the other figures.

remained invariant within the errors (Fig. 3). However, variations of $\sim 30 \%$ could be present in the data, comparable to the radio and optical variations.

\subsection{Optical and Near-Infrared Results}

\subsubsection{Photometry and Variability}

The near-infrared flux of PKS 2155-304 increased nearly monotonically by $\sim 0.2-0.3 \mathrm{mag}$ over a period of 7 days in all observed bands (Fig. 4), during the UV flaring period (Pian et al. 1997). The low $L$ magnitude is probably spurious and has much larger errors $(\sim 0.6 \mathrm{mag})$ than the other measurements. The dip seen in $H$ and $J$ may be real, but instrumental effects cannot be excluded.

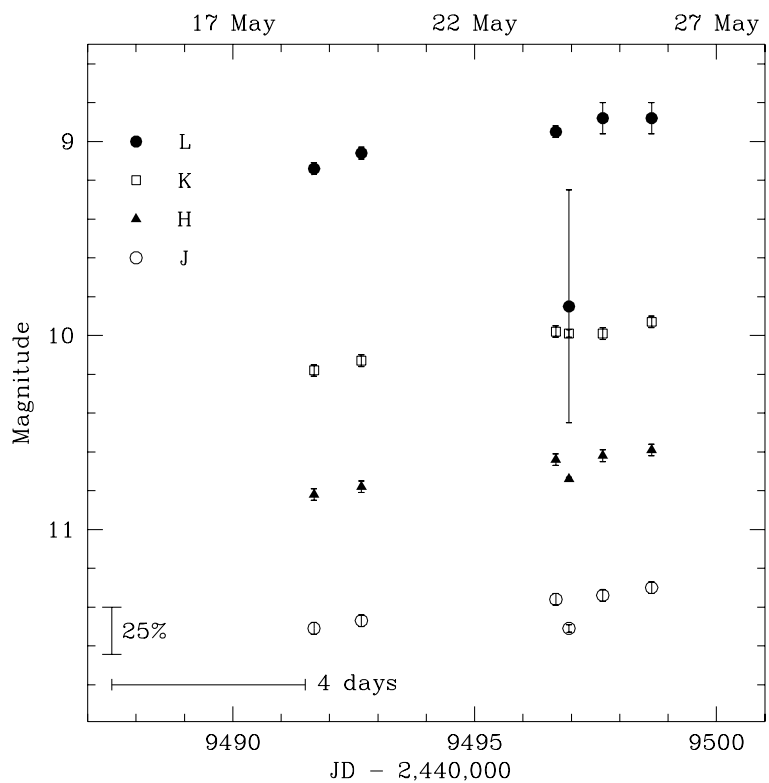

FIG. 4.- Near-infrared light curves from 1994 May. All bands increase by $\sim 0.3 \mathrm{mag}$ over the period of observations. The $L$ magnitude on MJD $9,496.95$ is probably spurious, whereas the dip seen in $H$ and $J$ on the same day may be real, but instrumental effects cannot be ruled out. A timescale bar is shown for comparison with the other figures. 


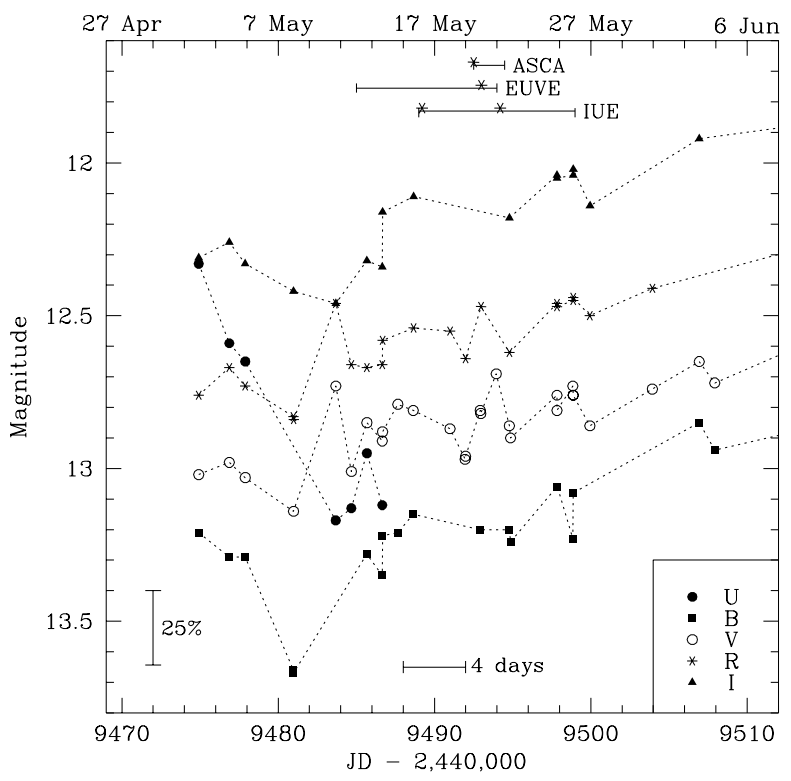

FIG. 5.-Optical light curves during the multiwavelength monitoring campaign, 1994 May. Where coverage is sufficient, it can be seen that all bands vary together. The $V$ - and $R$-band fluxes show a feature between MJD 9,492 and 9,495 that corresponds to the UV flare. Note the increase in $I$-band flux on $\sim 13$ May (MJD 9,486). This corresponds to a variation of $0.01 \mathrm{mag}$ minute ${ }^{-1}$. Uncertainties are the size of the points or smaller (they range from $\lesssim 0.01$ to $\sim 0.08 \mathrm{mag}$, with $0.01 \mathrm{mag}$ being typical). The durations of the $A S C A, E U V E$, and IUE experiments are shown at the top of the figure, and the midpoints of the flares are indicated with asterisks. A timescale bar is shown for comparison with the other figures. The dotted lines have been added to guide the eye only.

Figure 5 shows the optical (UBVRI) light curves for PKS 2155-304 during 1994 May. The durations of the X-ray, extreme-ultraviolet, and UV campaigns ( $A S C A, E U V E$, and $I U E$ ) are shown at the top (the middle of each flare is also indicated). While the coverage is sparse, general trends are the same in all wave bands. The sharp increase of 0.3 mag in the $V$-band flux, and to a lesser extent in the $R$-band flux, between MJD 9,492 and 9,494 corresponds to the flare seen in the UV at the same period (Urry et al. 1997). This increase does not seem present in the $B$ band (unless earlier), and there is no simultaneous data in the $U$ or $I$ band.

The entire April-November light curve for PKS 2155-304 is shown in Figure 6. There is a general "flickering" (miniflares of $\sim 0.2-0.4 \mathrm{mag}$ in several days) of the source in all bands throughout this period, superposed on a general, slow brightening (0.4-0.7 mag) through September, followed by a $0.4-0.7$ mag drop between the last observations in September (MJD 9,608) and the final observations in November (MJD 9,672). Throughout the observation period, PKS 2155-304 was brighter than the average $(B=13.58)$ seen by Pica et al. (1988) over the period 1979-1986. In early June (MJD 9,500-9,520), a large flare is seen in all observed optical bands. The amplitude of this feature is $0.3-0.4 \mathrm{mag}$, with a rise time of about 10 days and a duration of about 20 days, although it may not be resolved.

The largest observed excursions are a drop in the $B$ band of $\sim 0.5 \mathrm{mag}$ in 4 days and almost $1 \mathrm{mag}$ in $U$ in about 10 days, both in May, right at the start of the multiwavelength observing campaign (MJD 9,475-9,484; Fig. 5). The other bands exhibit a drop in magnitude over this period, but of much smaller amplitudes $(\sim 0.2 \mathrm{mag})$. There are no UV or $\mathrm{X}$-ray data during this period; the drop in flux in the 4.8



FIG. 6.-Complete optical light curves from 1994 April to November. Variations, from short-scale flickering ( $\sim 0.2 \mathrm{mag}$ in several days) to the longer term trends, are of similar amplitude in all wave bands, with no measurable lags. Uncertainties are the size of the points or smaller (they range from $\lesssim 0.01$ to $\sim 0.08 \mathrm{mag}$, with $0.01 \mathrm{mag}$ being typical). A timescale bar is shown for comparison with the other figures. The dotted lines have been added to guide the eye only.

$\mathrm{GHz}$ band is possibly correlated with the drop in the $U$ band, though this is most likely coincidental.

The fastest variations are changes of $0.1-0.2 \mathrm{mag}$ in tens of minutes. An example of this is the $I$-band flux at MJD $\sim 9,486$ (Fig. 5), which increases by 0.18 mag in 13.5 minutes ( $\sim 0.01$ mag minute ${ }^{-1}$ !), corresponding to a doubling time of 75 minutes; a lesser increase is also seen in the $B$ and $R$ bands. Several such increases are seen in the other bands over the observation period. In fact, if real, these are the fastest optical variations seen for any BL Lac object (by about a factor of 5; for OQ 530, Carini, Miller, \& Goodrich 1990 observed an increase of 0.06 mag in 20 minutes). A timescale of about an hour is consistent with the results of a structure analysis of several nights of photometry by Paltani et al. (1996), who found that the minimum timescale of variations is shorter than 15 minutes. The other variations that we observe, $\sim 0.01 \mathrm{mag} \mathrm{hr}^{-1}$ or several tenths of a magnitude over days, have been seen before in PKS 2155-304 (Carini \& Miller 1992) and are typical for these objects (e.g., BL Lac, OJ 287, Miller, Carini, \& Goodrich 1989; Carini et al. 1992; PKS 0235+164, Rabbette et al. 1996; S5 0716 + 714, Wagner et al. 1996). The blazar 3C 279, the subject of a similar multiwavelength campaign, was seen to double its $R$-band flux in 10 days (Grandi et al. 1996).

The most rapid variations observed yield a minimum doubling time or variability timescale, $\tau_{d}=75$ minutes. This, in fact, may not be a doubling timescale since we have not observed a true doubling of the flux. Nonetheless, we can estimate an upper limit to the black hole mass if we assume that these variations are caused by radiation generated close to a supermassive black hole (at $R=3 R_{\mathrm{s}}$, where $R_{\mathrm{S}}=2 G M / c^{2}$ is the Schwarzschild radius) and that the emission is isotropic. An estimate of the upper limit to the size of the emitting region is $R \approx \delta c \tau_{d}$, where $\delta$ is the Doppler factor, which takes relativistic beaming into account; $\delta \sim 10$. 
The limit to the mass of the black hole can be estimated by

$$
M_{\mathrm{var}} \approx \frac{R c^{2}}{6 G} \lesssim \frac{\delta c^{3} \tau_{d}}{6 G}
$$

For PKS $2155-304$ we calculate $M_{\text {var }} \lesssim 1.5 \times 10^{9}(\delta / 10)$ $M_{\odot}$, consistent with constraints based on UV and X-ray observations (Morini et al. 1986; Urry et al. 1993) and considerably smaller than what was found by Carini \& Miller (1992), taking into account the relativistic-beaming term.

\subsubsection{Colors and Spectral Shape}

In general, the optical light curves of PKS 2155-304 track each other well. No lags are detected, although because of poor sampling we may be insensitive to lags of several days in many cases. The $B-V$ and $V-I$ colors of PKS 2155-304 were calculated from simultaneous or nearly simultaneous measurements (the majority are within 1-5 minutes, and eight are within 10-40 minutes). During 1994 May, the $B-V$ colors varied from 0.2 to $0.5 \mathrm{mag}$, but for most of the rest of the observation period, they were near the average value of $\langle B-V\rangle=0.32 \pm 0.02 \mathrm{mag}$. Except for three points in early May, the $V-I$ colors were nearly constant at $\langle V-I\rangle=0.69 \pm 0.01 \mathrm{mag}$ (Fig. 7, top).

The largest color variations occurred when PKS 2155-304 was faint $(V \gtrsim 12.7)$. For $V<12.7$, the standard deviation of the $B-V$ colors is $0.003 \mathrm{mag}$, while for $V>12.7$ it is $0.03 \mathrm{mag}$ (Fig. 7, bottom). This is not due to increased measurement errors when the source fades, since the average errors in the ranges $V<12.7$ and $V>12.7$ are the same. The colors are constant except for observations before MJD 9500, at the start of the campaign, and at MJD 9,672, at the end, when the source was faint $(\langle V\rangle=12.87 \pm 0.11 \quad$ mag, compared with $\langle V\rangle=12.55 \pm 0.19$ mag at the other times).

The 1994 June 3 (MJD 9,506.9875) Lick spectrum of PKS 2155-304 is shown in Figure 8. The good data cover the
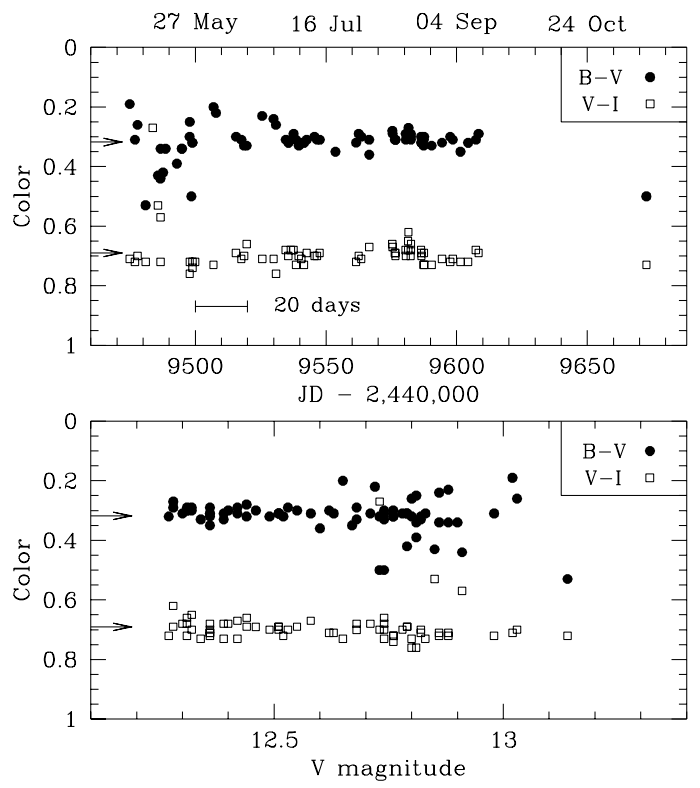

FIG. 7.- $B-V$ (circles) and $V-I$ (squares) colors for PKS $2155-304$ as a function of time (top) and $V$ magnitude (bottom). The largest color variations occur when the source is faint $(V \gtrsim 12.7)$. Average colors are $\langle B-V\rangle=0.32 \pm 0.02 \mathrm{mag}$ and $\langle V-I\rangle=0.69 \pm 0.01 \mathrm{mag}$ and are marked with arrows. The magnitudes used to calculate the colors were obtained simultaneously or nearly so (within $\lesssim 10$ minutes in most cases).

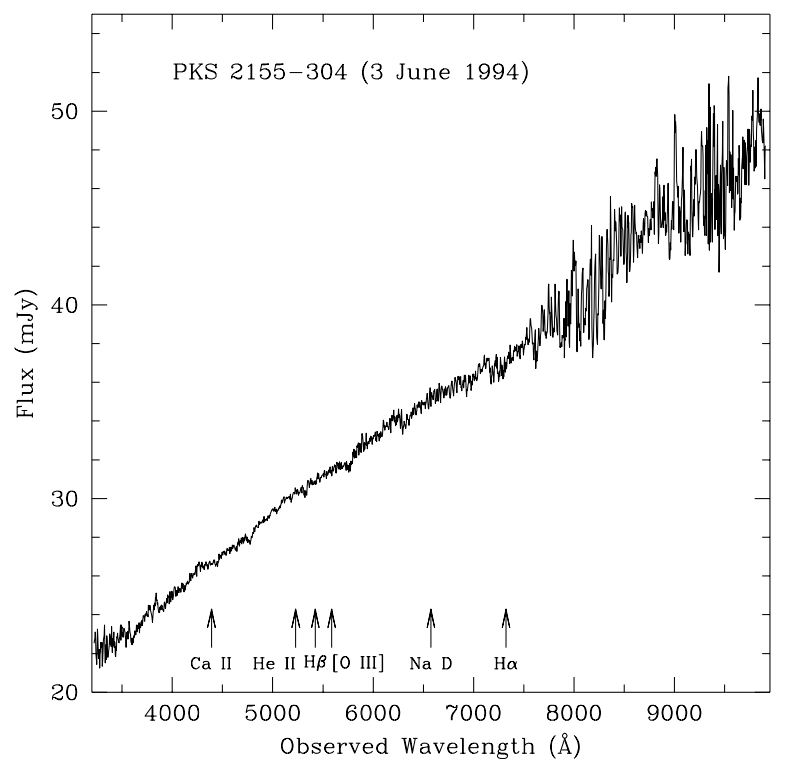

FIG. 8.-Optical spectrum of PKS 2155-304 from Lick Observatory (MJD 9,506.9875). A power law with index $\alpha=-0.71 \pm 0.02$ (where $F_{v} \propto$ $\left.v^{\alpha}\right)$ is a good representation of the spectrum, which is featureless to an equivalent width limit of $\sim 1 \AA$ (or even $0.5 \AA$ in most places). Typical features, if present at $z=0.116$, would be at the locations marked. The high-frequency oscillations most noticeable redward of $7500 \AA$ are produced by incompletely flattened CCD interference fringes.

wavelength range 4000-7500 $\AA$. Excessive noise in the region redward of $\sim 7600 \AA$ is an artifact of the highamplitude interference fringes produced by the CCD; division by flat fields did not remove them completely, because of flexure of the spectrograph. Several weak features are visible in the optical region, but these are likely to be calibration errors; there appear to be no unambiguous absorption or emission lines to an equivalent width limit of $\sim 1 \AA$, and perhaps even $0.5 \AA$ at most locations. Features typically observed in the spectra of these objects, if present, would be found at the locations marked (assuming $z=0.116$; Falomo, Pesce, \& Treves 1993). A power law of index $\alpha=-0.71 \pm 0.02$ (where $F_{v} \propto v^{\alpha}$ ) provides a good fit to the spectrum. This is identical to the power-law index derived by Courvoisier et al. (1995) from Lick spectra of PKS 2155 - 304 obtained on 1991 October 31 and December 14.

As a further check of the continuum shape, we converted simultaneous magnitudes (mostly $U B V R I$, covering the range $\sim 3600-9000 \AA$ ) to fluxes by using zero points from Bessell (1979). We then fitted the continuum with a power law (as above) to obtain individual spectral slopes, and we find an average $\left\langle\alpha_{U B V R I}\right\rangle=-0.76 \pm 0.03$ (Fig. 9, top). This is consistent with what we found from the Lick spectrum presented here and with what was found in previous studies (Smith \& Sitko 1991; Smith et al. 1992; Courvoisier et al. 1995; Paltani et al. 1996). The spectra are slightly steeper during the period when $V>12.7$ (Fig. 9, bottom).

\subsubsection{Polarization}

As with the 1991 November multiwavelength monitoring campaign (Smith et al. 1992), the optical polarization exhibited strong variability during 1994 May. The degree of linear polarization $(P)$ ranged from $\sim 3 \%$ to $\sim 14 \%$ (Table 8 and Fig. 10, top), and the polarization position angle $(\theta)$ varied from $\sim 100^{\circ}$ to $\sim 150^{\circ}$ (Fig. 10, bottom). Indeed, a change in $\theta$ of nearly $25^{\circ}$ was observed between May 15 and 17 (MJD 9,487.96 and 9,489.95). 

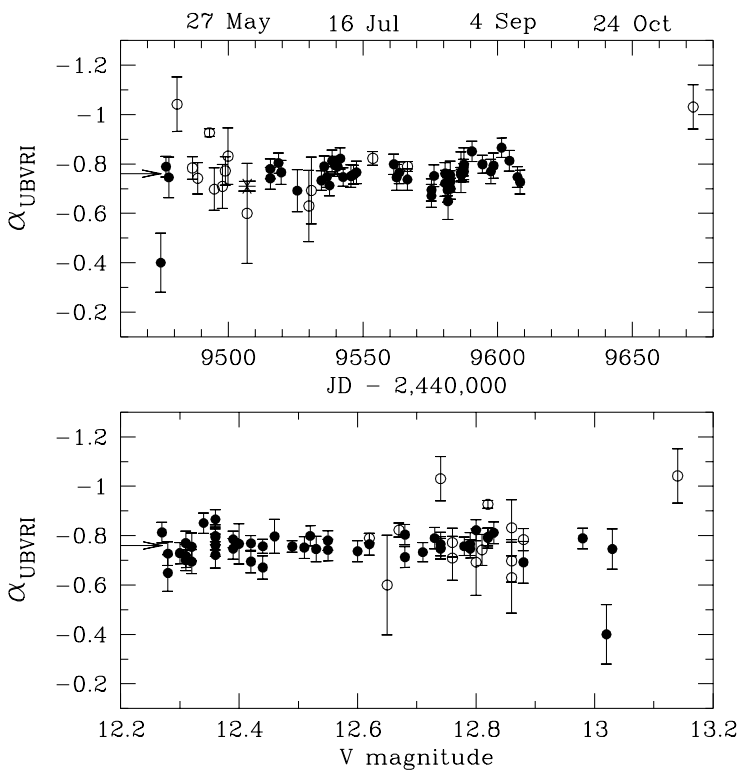

FIG. 9.-Top: Slope of the total flux energy distribution derived from fits $\left(F_{v} \propto v^{\alpha}\right)$ to five simultaneous $U B V R I$ measurements ( filled circles) and three or four simultaneous measurements (open circles). The average slope is $\left\langle\alpha_{U B V R I}\right\rangle=-0.76 \pm 0.03$ (arrow). The asterisk is the slope from the Lick spectrum. Bottom: Same as above, but as a function of $V$-band magnitude. There is a very slight steepening of the spectrum with increasing magnitude, although this is not significant.

Broadband $U B V R I$ polarimetry acquired on May 19-21 (MJD 9,491.96-9,493.94) shows the development of strong wavelength-dependent polarization. Since only $V$-band measurements were made prior to May 19 (MJD 9,492), it is impossible to know how $P$ and/or $\theta$ changed with wave-

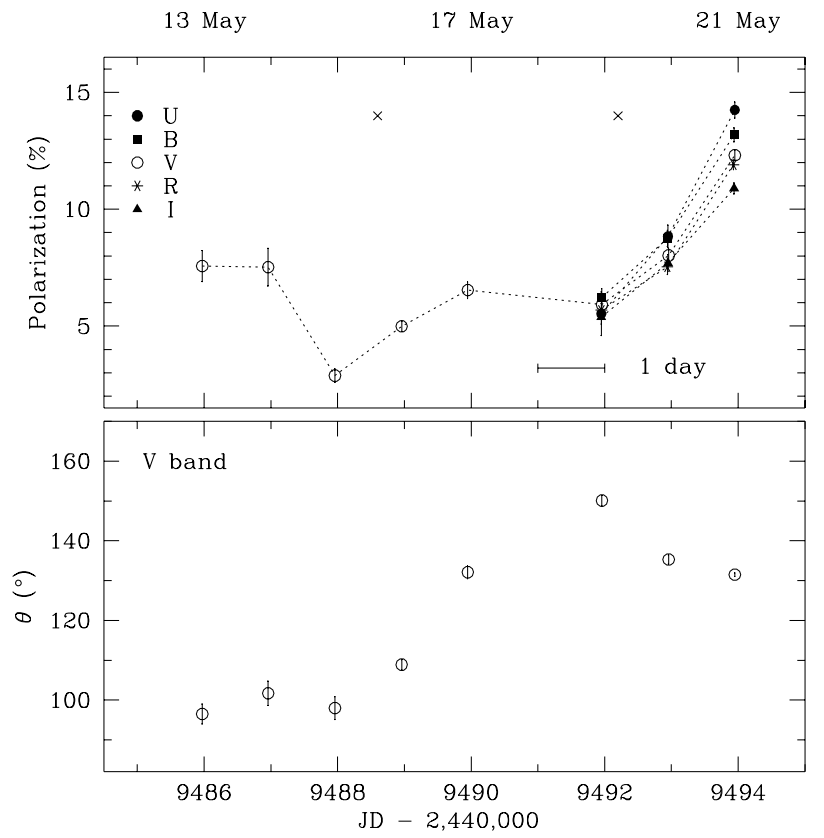

Fig. 10.-Top: Polarization light curves from 1994 May (Mount Lemmon, Arizona). The increase in polarization after MJD 9,492 occurs in all wave bands, and the wavelength-dependent polarization is obvious. The increases in polarization after MJD 9,488 and 9,492 occur at the same time as the UV flaring events, the start times of which are marked with crosses (Urry et al. 1997). A timescale bar is shown for comparison with the other figures. The dotted lines have been added to guide the eye only. Bottom: Polarization position angle for the $V$ band. The preferred range is $\sim 90^{\circ}-150^{\circ}$. length during this period. However, it is apparent that any wavelength dependence was weak on May $19\left(P_{U} / P_{I}=1.03\right.$ \pm 0.19 ), while on May 20 (MJD 9,493) $P$ clearly increases toward the blue $\left(P_{U} / P_{I}=1.15 \pm 0.08\right)$. Strong wavelength dependence is observed on the following night, with $P_{U} / P_{I}=1.31 \pm 0.04$. Though the position angle exhibits no trend, the dependence upon wavelength of $P$ is among the strongest ever observed for PKS 2155-304, and we note that on May 21 (MJD 9,493.9) the $U$-polarization $(14.3 \%)$ is the highest optical polarization reported for this object (cf. Smith et al. 1992). The increases in polarization after May 15 and 19 (MJD 9,487.96 and 9,491.96) correspond to the two UV flaring events (Urry et al. 1997).

Figure 11 shows the polarized $V$-band flux as a function of the $V$-band flux, ordered chronologically. Except for points $6-8$, the optical photometry and polarization were not strictly simultaneous; there are differences of about $7 \mathrm{hr}$ between measurements for points 1-4 and 1 day for point 5 . There are no definite trends, and in fact, PKS 2155-304 becomes both brighter and fainter as the polarization increases. The UV flaring events occur after observations 3 and 6.

Polarization observations later in the year (Table 9) show a general decrease from about $10 \%$ in July to around $5 \%$ in October (for the $B$ and $V$ bands, at least). At the same time, the object was brightening in all optical bands. It is interesting to note that the polarization position angle shows no preferred trend with percent polarization; for these observations it decreases with decreasing polarization, while in May it both increased and decreased with increasing polarization.

However, the observed position angles of PKS 2155-304 have been mostly confined between about $90^{\circ}$ and $150^{\circ}$ (Smith et al. 1992; Allen et al. 1993; Jannuzi, Smith, \& Elston 1993). This has also been the case for the data collected during the period covered in this paper

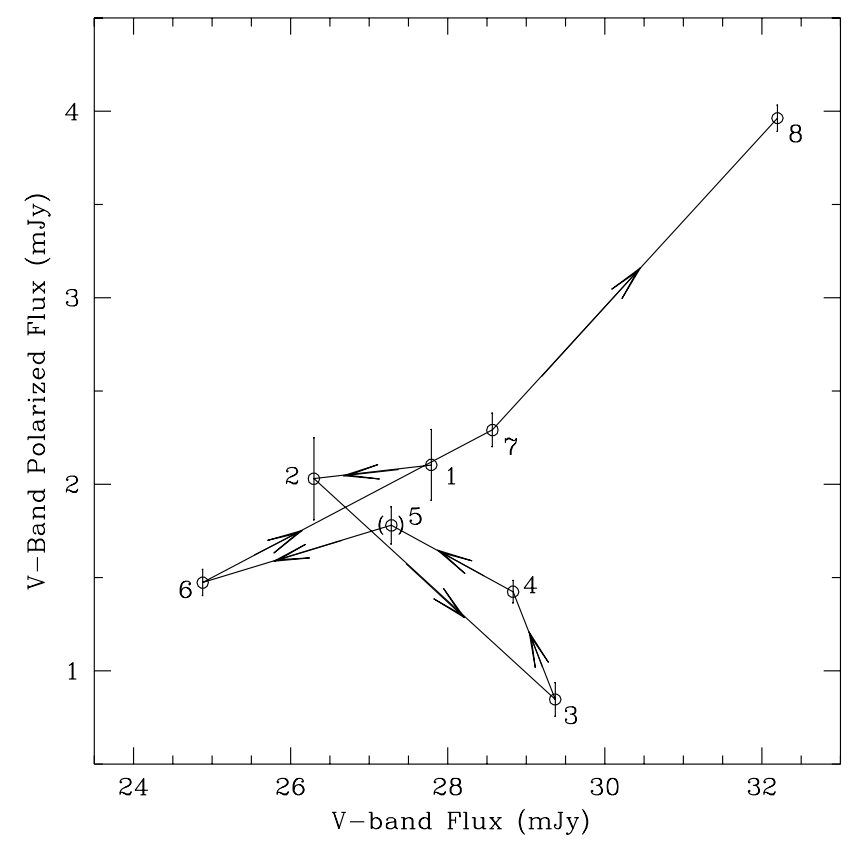

Fig. 11.-Polarized $V$-band flux vs. $V$-band flux for 1994 May, numbered in chronological order. PKS 2155-304 both brightens and fades when the polarization increases. The two UV flaring events occurred after observations 3 and 6 . 
(Tables 8 and 9; Fig. 10, bottom), in contrast to, for example, BL Lac itself (Moore et al. 1982).

The preferred polarization orientation for PKS 2155-304 may indicate that the line of sight to PKS $2155-304$ is not as close to the symmetry axis as may be the case for BL Lac. These two examples also reflect the general difference between X-ray-selected BL Lac objects (like PKS 2155-304) and radio-selected BL Lac objects (like BL Lac itself), in that X-ray-selected objects have preferred position angles more often than radio-selected ones (Jannuzi, Smith, \& Elston 1994).

The USP CCD imaging polarimetry also allowed measurements of the foreground stars in the field of PKS 2155-304. From $V$-filter images taken on July 2, we selected seven stars.

A weighted average of the measured $Q / I$ and $U / I$ Stokes parameters yielded $P=0.31 \% \pm 0.03 \%$ at 114.5 . In our fields, star 5 of Hamuy \& Maza (1989) shows $P=0.27 \% \pm 0.04 \%$ at 121.1 , in excellent agreement with the field average. This corroborates earlier findings (Courvoisier et al. 1995) that the interstellar polarization toward PKS 2155-304 is negligible.

\section{CONCLUSIONS}

In 1994 May, the bright BL Lac object PKS 2155-304 was the subject of a large multiwavelength campaign. In this paper, we presented the ground-based radio, near-infrared, and optical results of the campaign, along with additional observations made throughout the year to 1994 November.

The $8.4,15$, and $22.5 \mathrm{GHz}$ data seem to vary together over the observation period. There is possibly a lag of several days between the 8.4 and $15 \mathrm{GHz}$ data and those at $22.5 \mathrm{GHz}$. When compared with the optical data obtained over the same period, there is no direct correlation, although the $4.8 \mathrm{GHz}$ data from the first 10 days of observations may correlate with the optical data, with no measured lags. Any correlation between the radio and the optical data could be spurious, however, since there are variations on timescales of several days in both wave bands and many large gaps in coverage.

The millimeter data are essentially invariant, although variations of $\sim 30 \%$ are possible within the large errors. The near-infrared points exhibit a monotonic increase in brightness over their short observation period. Both these data sets show variations comparable to what is seen in the optical and radio, and there are no apparent correlations. The light curves in the optical bandpasses vary together and show similar short- and long-scale characteristics throughout the observation period. The fastest variations, of 0.01 mag minute ${ }^{-1}$, make PKS $2155-304$ the most optically rapidly variable BL Lac observed to date and are similar to timescales observed in the UV (Pian et al. 1997). More typically, variations are $\sim 0.01 \mathrm{mag} \mathrm{hr}^{-1}$, or several tenths of a magnitude over days, which is what is seen for other blazars (see, e.g., Carini \& Miller 1992). With a large number of assumptions, we limit the mass of the central black hole to $M_{\text {var }} \lesssim 1.5 \times 10^{9}(\delta / 10) M_{\odot}$; this is consistent with the mass determined from UV and X-ray constraints, and considerably less than what was determined previously in the optical band.

Smith et al. (1992) and Courvoisier et al. (1995) found the source to have constant color. Trends in $B-V$ color have been noted before, in the sense of bluer $B-V$ as the source fades (Miller \& McAlister 1983; Carini \& Miller 1992), but during the present campaign the opposite occurred, with slightly redder colors as the source faded, similar to what was found by Treves et al. (1989) and Smith \& Sitko (1991). The effect is small, however. The optical fluxes tracked each other well, indicating that intensive, multiband optical monitoring is not necessary for such campaigns. Instead, the object can be observed several times per night in all bands, but intensively in just two or three.

Polarimetry measurements showed marked color dependence of the polarization (higher polarization toward the blue), in fact, the strongest such dependence ever observed for PKS 2155-304. The object was also seen to have the highest optical polarization observed $(U=14.3 \%)$, although in the range typical for X-ray-selected BL Lac objects (Jannuzi et al. 1994). Also typical for X-ray-selected objects is the preferred position angle of the polarization that we observed for PKS 2155-304.

J. E. P., E. P., and C. M. U. would like to acknowledge support from NASA grants NAG 5-1918, NAG 5-1034, and NAG 5-2499. H. D. A. and M. F. A. acknowledge support from NSF grant AST 94-21979, A. V. F. from NSF grant AST 89-57063, E. J. H. from NASA grant NGT-51152, R. I. K. and C. P. from NASA Long-Term Space Astrophysics grant NAGW-2120, and P. S. S. from NASA grant NAG 5-1630. M. D. J. would like to thank the Brigham Young University Department of Physics and Astronomy for continued support of his research. A. M. M. and C. V. R. received support from the São Paulo state funding agency FAPESP through grant 94/0033-3. The University of Michigan Radio Astronomy Observatory is supported by the National Science Foundation and by funds from the University of Michigan. Sergio Ortolani is thanked for providing data. Some observations were obtained in the service observing mode from the Jacobus Kapteyn Telescope on La Palma; the help of Vik Dhillon, Derek Jones, Reynier Peletier, and Keith Tritton and the two service observers, Phil Rudd and Emilios Harlaftis, is greatly appreciated. The SAAO CCD data (D. A. H. B.) were obtained using a focal reducer provided by $\mathrm{M}$. Shara (STScI). This research made use of the NASA/IPAC Extragalactic Database (NED), operated by the Jet Propulsion Laboratory, Caltech, under contract with NASA, and of NASA's Astrophysics Data System abstract service (ADS).

\section{REFERENCES}

Allen, R. G., Smith, P. S., Angel, J. R. P., Miller, B. W., Anderson, S. F., \& Margon, B. 1993, ApJ, 403, 610

Aller, H. D., Aller, M. F., Latimer, G. E., \& Hodge, P. E. 1985, ApJS, 59, 513

Baars, J. M. W., Genzel, R., Pauliny-Toth, I. I. K., \& Witzel, A. 1977, A\&A, 61,99

Bessell, M. S. 1979, PASP, 91, 589

Blandford, R. D., \& Rees, M. J. 1978, in Pittsburgh Conf. on BL Lac Objects, ed. A. M. Wolfe (Pittsburgh: Univ. Pittsburgh Dept. Phys. Astron.), 328

Booth, R. S., Delgado, G., Hagstrom, M., Johansson, L. E. B., \& Murphy, D. C. 1989 , A\&A, 216, 315

Bregman, J. N., \& Hufnagel, B. 1989, in Lecture Notes in Physics, 334, BL Lac Objects, ed. L. Maraschi, T. Maccacaro, \& M.-H. Ulrich (Berlin: Springer), 150

Brinkmann, W., et al. 1994, A\&A, 288, 433

Carini, M. T., \& Miller, H. R. 1992, ApJ, 385, 146

Carini, M. T., Miller, H. R., \& Goodrich, B. D. 1990, AJ, 100, 347

Carini, M. T., Miller, H. R., Noble, J. C., \& Goodrich, B. D. 1992, AJ, 104, 15 
Carter, B. S. 1990, MNRAS, 242, 1

Courvoisier, T. J.-L., et al. 1995, ApJ, 438, 108

Edelson, R. A., et al. 1995, ApJ, 438, 120

Falomo, R., Pesce, J. E., \& Treves, A. 1993, ApJ, 411, L63

Frater, R. H., Brooks, J. W., \& Whiteoak, J. B. 1992, J. Electrical Electron. Eng. Australia, 12, 103

Fruscione, A., Bowyer, S., Königl, A., \& Kahn, S. 1994, ApJ, 422, L55

Ghisellini, G., Maraschi, L., \& Treves, A. 1985, A\&A, 146, 204

Grandi, P., et al. 1996, ApJ, 459, 73

Hamuy, M., \& Maza, J. 1989, AJ, 97, 720

Hughes, P. A., Aller, H. D., \& Aller, M. F. 1989, ApJ, 341, 68

Jannuzi, B. T., Smith, P. S., \& Elston, R. 1993, ApJS, 85, 265 1994, ApJ, 428, 130

Kii, T., et al. 1997, in preparation

Königl, A. 1989, in Lecture Notes in Physics, 334, BL Lac Objects, ed. L. Maraschi, T. Maccacaro, \& M.-H. Ulrich (Berlin: Springer), 321

Magalhães, A. M., Rodrigues, C. V., Margoniner, V. E., Pereyra, A., \& Heathcote, S. 1996, in ASP Conf. Ser. 97, Polarimetry of the Interstellar Medium, ed. D. C. B. Whittet \& W. G. Roberge (San Francisco: ASP), 118

Marscher, A. P., \& Gear, W. K. 1985, ApJ, 298, 114

Marshall, H. L., Carone, T. E., \& Fruscione, A. 1993, ApJ, 414, L53

Marshall, H. L., et al. 1997, in preparation

Massey, P., Strobel, K., Barnes, J. V., \& Anderson, E. 1988, ApJ, 328, 315
Miller, H. R., Carini, M. T., \& Goodrich, B. D. 1989, Nature, 337, 627

Miller, H. R., \& McAlister, H. A. 1983, ApJ, 272, 26

Miller, J. S., \& Stone, R. P. S. 1993, Lick Obs. Tech. Rep., No. 66

Moore, R. L., et al. 1982, ApJ, 260, 415

Morini, M., Chiappetti, L., Maccagni, D., Maraschi, L., Molteni, D., Tanzi, E. G., Treves, A., \& Wolter, A. 1986, ApJ, 306, L71

Oke, J. B., \& Gunn, J. E. 1983, ApJ, 266, 713

Padovani, P., \& Giommi, P. 1995, ApJ, 444, 567

Paltani, S., Courvoisier, T. J.-L., Bratschi, P., \& Blecha, A. 1996, in ASP Conf. Ser. 110, Blazar Continuum Variability, ed. H. R. Miller, J. R. Webb, \& J. C. Noble (San Francisco: ASP), 36

Pian, E., et al. 1997, ApJ, 486, 784

Pica, A. J., Smith, A. G., Webb, J. R., Leacock, R. J., Clements, S., \& Gombola, P. P. 1988, AJ, 96, 1215

Rabbette, M., McBreen, B., Steel, S., \& Smith, N. 1996, A\&A, 310, 1

Smith, P. S., Hall, P. B., Allen, R. G., \& Sitko, M. L. 1992, ApJ, 400, 115

Smith, P. S., Jannuzi, B. T., \& Elston, R. 1991, ApJS, 77, 67

Smith, P. S., \& Sitko, M. L. 1991, ApJ, 383, 580

Treves, A., et al. 1989, ApJ, 341, 733

Urry, C. M., et al. 1993, ApJ, 411, 614 1997, ApJ, 486, 799

Vestrand, W. T., Stacy, J. C., \& Sreekumar, P. 1995, ApJ, 454, L93

Wagner, S. J., \& Witzel, A. 1995, ARA\&A, 33, 163

Wagner, S. J., et al. 1996, AJ, 111, 2187 